\title{
Fully-dynamic risk-indifference pricing and no-good-deal bounds
}

\author{
Jocelyne Bion-Nadal* and Giulia Di Nunno ${ }^{\dagger \ddagger}$
}

February $10^{\text {th }}, 2020$

\begin{abstract}
The seller's risk-indifference price evaluation is studied. We propose a dynamic risk-indifference pricing criteria derived from fully-dynamic risk measures on the $L_{p}$-spaces for $p \in[1, \infty]$. The concept of fullydynamic risk measures extends the one of dynamic risk measures by adding the actual possibility of changing the risk perspectives over time. This family is then characterised by a double time index. Our framework fits well the study of both short and long term investments. In this paper we analyse whether the risk-indifference pricing criterion actually provides a proper convex price system. It turns out that, depending on $p$, this is not always the case. Then it becomes necessary and extension of the framework beyond $L_{p}$.

Furthermore, we consider the relationship of the fully-dynamic riskindifference price with no-good-deal bounds. We shall provide necessary and sufficient conditions on the fully-dynamic risk measures so that the corresponding risk-indifference prices satisfy the no-good-deal bounds. Remarkably, the use of no-good-deal bounds also provides a method to select the risk measures and thus construct a proper fullydynamic risk-indifference price system within the $L_{2}$-spaces.

Key-words: convex prices, risk-indifference prices, time-consistency, extension theorems, dynamic risk measures, no-good-deal.
\end{abstract}

\section{Introduction}

Risk-indifference pricing was proposed as an alternative to utility indifference, as a pricing approach in incomplete markets, see e.g. [43]. The idea is to replace the performance of a portfolio described in terms of the agent's utility function with the performance based on the risk exposure measured

${ }^{*}$ UMR 7641 CNRS - Ecole Polytechnique. Ecole Polytechnique, 91128 Palaiseau Cedex, France. Email: jocelyne.bion-nadal@cmap.polytechnique.fr

${ }^{\dagger}$ Department of Mathematics, University of Oslo, P.O. Box 1053 Blindern, N-0316 Oslo Norway. Email: giulian@math.uio.no

${ }^{\ddagger}$ Norwegian School of Economics (NHH), Helleveien 30, N-5045 Bergen, Norway. 
by convex risk measures. Indeed, instead of maximising the utility, which is often quite difficult for a trader to write down explicitly, the risk-indifference criterion minimises the risk exposure. The point of connection between the two approaches is represented by the entropic risk measure which corresponds to an exponential-type utility. The price of a financial claim is then defined as the amount such that the risk exposure remains the same whether the optimal trading strategy includes the claim or not.

This pricing approach was introduced on a fixed time horizon $T$ characterised by the expiration time of the financial claim $X$. The corresponding price $x_{0 T}(X)$ is evaluated at the present time $s=0$. A large part of the literature has focused on the stochastic control techniques necessary to compute the price $x_{0 T}(X)$ of $X$. The different techniques depend on the choice of risk measure, on the market dynamics of the fundamental traded underlying assets, and on the information flow available. See e.g. [25, 29, 39]. Again depending on the underlying price dynamics and the information flow available, one can extend some of these results to obtain the price evaluation for any time $s \in[0, T]$.

On the other side, [33] presents a systematic study of the risk-indifference price system $\left(x_{s T}(X)\right)_{s}$ for essentially bounded claims $X \in L_{\infty}\left(\mathcal{F}_{T}\right)$. Similar to the present paper, also [33] is kept free of the particular choice of underlying price dynamics.

In [33] the evaluation of each price $x_{s T}(X)$ is linked with one element $\rho_{s}$ from the dynamic convex risk measure $\left(\rho_{s}\right)_{s}$. The risk measure $\rho_{s}$ evaluates the risks at time $s$ of any financial position with expiration $t \in(s, T]$ as if it was a risk occurring at time $T$. However, particularly thinking of long time horizons, it seems reasonable to allow for the possibility of modelling changes in the risk evaluation criteria. For this reason, in this paper, we consider a fully-dynamic convex risk measure, namely a family of risk measures indexed by two points in time $\left(\rho_{s t}\right)_{s, t}$. So working with fully-dynamic risk measures has important consequences from the modelling point of view.

As we shall see, the use of fully dynamic risk measures extends and clarifies the discussion on the concepts of time-consistency with respect to risk evaluations, bringing insight also from the point of view of mathematical analysis. To explain briefly, the notion of strong time-consistency for a fully-dynamic risk measure $\left(\rho_{s t}\right)_{s, t}$ was introduced in [9] and there also completely characterised. In the present work, we introduce the concept of time-consistency for the fully-dynamic risk measure $\left(\rho_{s t}\right)_{s, t}$ (see Definition 2.10). Both concepts are compared with the meaning of time-consistency for the dynamic risk measure $\left(\rho_{s}\right)_{s}$, up to now mostly frequently used in the literature, see e.g. [1]. We find out that the relationship among all these concepts is delicate (see Proposition 2.13 and Corollary 2.14) and leads to appreciate further the difference between fully-dynamic and dynamic risk measures.

Hereafter we give an overview of the structure of the present work, high- 
lighting the main results. The paper is organised in two main parts, each divided in subsections.

Section 2 deals with risk-indifference pricing. After the formal introduction of the framework of convex price operators and convex price systems, we proceed by presenting the risk-indifference price operators, which are defined via risk measures. Note that we work with fully-dynamic risk measures $\left(\rho_{s t}\right)_{s, t}$ assumed to be strong time-consistent, but not to be a priori normalised.

In Subection 2.1 the relevant concepts about $\left(\rho_{s t}\right)_{s, t}$ are introduced. In particular we study the different concepts of time-consistency as well as the relationship between fully-dynamic and dynamic risk measures. Furthermore, the two concepts of a dominated and a sensitive risk measure are introduced with the corresponding results. The subsection is completed presenting examples of strong time-consistent fully-dynamic risk measures.

Subsection 2.2 deals with risk-indifferent pricing. The price operator $x_{s t}$ is given by means of $\rho_{s t}$ belonging to the strong time-consistent fully-dynamic risk measure $\left(\rho_{s t}\right)_{s, t}$. We study the properties of $x_{s t}$ to see if the riskindifference price is indeed a convex price operator. Actually, we find that, for $p \in[1, \infty)$ under mild assumptions, a risk-indifference price operator $x_{s t}$ is a mapping from $L_{p}\left(\mathcal{F}_{t}\right)$ into the space of $\mathcal{F}_{s}$-measurable random variables, but the operator takes its values in $L_{p}\left(\mathcal{F}_{s}\right)$ only on a restricted domain $L_{t}:=\operatorname{Dom} x_{s t} \subsetneq L_{p}\left(\mathcal{F}_{t}\right)$. On the contrary, if $p=\infty$, the operator $x_{s t}$ is well-defined on $L_{\infty}\left(\mathcal{F}_{t}\right)$ with values in $L_{\infty}\left(\mathcal{F}_{s}\right)$. Because of this, the study of the Fatou property (and of the time-consistency for the prices $\left(x_{s t}\right)_{s, t}$ ) is far from trivial when $p<\infty$.

We tackle the issue by extending the operator $x_{s t}$ from $\operatorname{Dom} x_{s t} \subsetneq L_{p}\left(\mathcal{F}_{t}\right)$ to an appropriate Banach space $L_{t}^{c}$. To achieve such an extension, which also should maintain the structure of a risk-indifference operator, we have first to extend the underlying risk measure on $L_{t}^{c}$. We stress that we have to perform the extension of the whole family of risk measures in a careful way, so that the new extended fully-dynamic risk measure is also strong timeconsistent. For this we make use of the notions of sensitive and dominated risk measures. The domination is inspired by sandwich extension theorems as in [14] and it is used in [15] to obtain a representation of risk measures. The sensitivity is linked to the relevance as introduced in [33] and it allows to write the representation of risk measures in terms of $P$-equivalent probability measures. See [28]. Once the risk measure $\rho_{s t}$ is extended, it is easy to derive an extension of the price $x_{s t}$ as a mapping defined on $L_{t}^{c}$ with values in $L_{s}^{c}$. This extension also fulfils the requirements to be a convex price operators. Here we emphasise that the use of strong time-consistency of $\left(\rho_{s t}\right)_{s, t}$ is crucial in obtaining the not-standard representations of the risk measures in Proposition 2.46, which are in their turn a crucial element for 
the arguments proposed later. Also, it is the strong time-consistency that allows to assume sensitivity and dominatoion only on the risk measure $\rho_{0 T}$ so to obtain the above mentioned representations and subsequent extensions (see Theorem 2.49).

Subsection 2.3 deals with the whole family of risk-indifference price operators $\left(x_{s t}\right)_{s, t}$ defined as mappings $x_{s t}$ on $L_{t}^{c}$ with values in $L_{s}^{c}$. We find that this family of operators $\left(x_{s t}\right)_{s, t}$ on $\left(L_{t}^{c}\right)_{t}$ is time-consistent and thus it has all the properties of a convex price system. By this we can conclude that dealing with risk indifference pricing from a dynamic perspective is actually a delicate affair, important fact to keep in mind when proceeding to actual computations. We complete the section showing that, for all $t$ and $X \in L_{t}^{c}$, the processes $x_{s t}(X), s \in[0, t]$, admit càdlàg modification. This also ensures that stopping times can be considered.

Working with the Banach spaces $\left(L_{t}^{c}\right)_{t}$ is mathematically effective, though not straightforward. For this reason in Section 3, we propose to constrain prices by no-good-deal bounds. This leads to a risk-indifference price system $\left(x_{s t}\right)_{s, t}$ with domain $\left(L_{2}\left(\mathcal{F}_{t}\right)\right)_{t}$ and not $\left(L_{t}^{c}\right)_{t}$. No-good-deal bounds have been introduced in a dynamic setting in [13]. Here we study first the representation of convex no-good-deal prices and then we combine this with the risk-indifference prices. Our final result gives a characterisation of those risk measures for which the risk-indifference prices are actually no-good-deal.

\section{Risk-indifference pricing}

In this section we first present the general framework of convex price systems, then we introduce fully-dynamic risk measures $\left(\rho_{s t}\right)_{s, t}$ and their properties. From these we define the risk-indifference price operators. Both the individual price operator and the whole family of these will be studied in detail to see whether they constitute a convex price system.

For the time horizon $[0, T]$, with $T \in(0, \infty)$, let $\left(\Omega, \mathcal{F},\left(\mathcal{F}_{t}\right)_{t}, P\right)$ be a complete filtered probability space. We assume that $\mathcal{F}_{0}$ is trivial. For any $p \in[1, \infty]$ and a $\sigma$-algebra $\mathcal{B}$ of events of $\Omega$, we consider the space $L_{p}(\mathcal{B}):=$ $L_{p}(\Omega, \mathcal{B}, P)$ of real valued random variables with the finite norms:

$$
\|X\|_{p}:= \begin{cases}\left(E\left[|X|^{p}\right]\right)^{1 / p}, & p \in[1, \infty) \\ \operatorname{esssup}|X|, & p=\infty\end{cases}
$$

We denote the expectation under $P$ by $E$. If other measures are used they will be specified. The claims and all financial positions belong to an $L_{p}$-space for $p \in[1, \infty]$. For any time $t \in[0, T]$, we consider the convex subset:

$$
L_{t} \subseteq L_{p}\left(\mathcal{F}_{t}\right): \quad L_{t} \subseteq L_{T} .
$$


The prices are in general defined on the domain $L_{t}$ representing the set of purchasable assets. Note that, in general, $L_{t} \subseteq L_{p}\left(\mathcal{F}_{t}\right)$ for some $t \in[0, T]$. In view of the financial application, it is justified to require that, for all $t$,

A1) $1 \in L_{t}$,

A2) $1_{A} X \in L_{t}$, for every $A \in \mathcal{F}_{t}$ and every $X \in L_{t}$.

To take care of the value of money over time, we consider a numéraire $R_{t}$, $t \in[0, T]$, used as discounting factor. This refers to a money market account. In this work we set $R_{t} \equiv 1$ to simplify the notation.

Definition 2.1. For any $s, t \in[0, T]$ with $s \leq t$, the operator

$$
x_{s t}: L_{t} \longrightarrow L_{s}
$$

is a (convex) price operator if it is:

- monotone, i.e. for any $X^{\prime}, X^{\prime \prime} \in L_{t}$ such that $X^{\prime} \geq X^{\prime \prime}$,

$$
x_{s t}\left(X^{\prime}\right) \geq x_{s t}\left(X^{\prime \prime}\right),
$$

- convex, i.e. for any $X^{\prime}, X^{\prime \prime} \in L_{t}$ and $\lambda \in[0,1]$,

$$
x_{s t}\left(\lambda X^{\prime}+(1-\lambda) X^{\prime \prime}\right) \leq \lambda x_{s t}\left(X^{\prime}\right)+(1-\lambda) x_{s t}\left(X^{\prime \prime}\right)
$$

- it has the Fatou property, i.e. for any $X \in L_{t}$ and any dominated sequence $\left(X_{n}\right)_{n}$ in $L_{t}$ (i.e. there exists $Y \in L_{p}\left(\mathcal{F}_{t}\right)$ such that $\left|X_{n}\right| \leq Y$ for all $n)$ which is converging $P$-a.s. to $X$, we have:

$$
\liminf _{n \rightarrow \infty} x_{s t}\left(X_{n}\right) \geq x_{s t}(X)
$$

- weak $\mathcal{F}_{s}$-homogeneous, i.e. for all $X \in L_{t}$

$$
x_{s t}\left(1_{A} X\right)=1_{A} x_{s t}(X), \quad A \in \mathcal{F}_{s},
$$

- projection property

$$
x_{s t}(X)=X, \quad X \in L_{p}\left(\mathcal{F}_{s}\right) \cap L_{t} .
$$

In particular we have $x_{s t}(0)=0, x_{s t}(1)=1$, and $x_{t t}(X)=X, X \in L_{t}$.

Note that the $\mathcal{F}_{S}$-homogeneity is sometimes called "locality" in the literature, see e.g. [7].

We remark that, if $p \in[1, \infty)$ and the operator $x_{s t}$ is monotone and linear (as in [2] and [23]), the weak $\mathcal{F}_{s}$-homogeneity is equivalent to $\mathcal{F}_{s}$-homogeneity, i.e. for all $X \in L_{t}, x_{s t}(\xi X)=\xi x_{s t}(X)$ for all $\xi \in L_{p}\left(\mathcal{F}_{s}\right)$ such that $\xi X \in L_{t}$. 
If $p=\infty$ and the operator is linear and semi-continuous, then the same result holds (see [13]).

Hereafter we introduce two concepts of time-consistency for the family of price operators. These concepts are specifically for the price operators and they are only to be compared, but not confused with those that will be given later for risk measures (cf. Definition 2.10).

Definition 2.2. The family of convex price operators $\left(x_{s t}\right)_{s, t}$ is

- strong time-consistent if, for all $r, s, t \in[0, T]: r \leq s \leq t$, we have that

$$
x_{r t}(X)=x_{r s}\left(x_{s t}(X)\right), \quad X \in L_{t},
$$

- time-consistent if, for all $t \in[0, T]$,

$$
x_{s t}(X) \geq x_{s t}(Y), \quad \text { for } X, Y \in L_{t},
$$

implies that

$$
x_{r t}(X) \geq x_{r t}(Y), \quad \text { for all } r \leq s \leq t .
$$

The notion of strong time-consistency is naturally extending the very property of linear prices, which comes from the chaining of conditional expectations. This notion is here distinguished from the time-consistency, which is a weaker notion in line with the literature of family of convex operators such as convex risk measures. We observe that the strong time-consistency of prices implies their time-consistency. This is easily derived by means of the projection property.

Definition 2.3. $A$ (convex) price system $\left(x_{s t}\right)_{s, t}$ on $\left(L_{t}\right)_{t}$ is the family of convex price operators (2.2) if it is at least time-consistent.

We study dynamic risk-indifference prices in the $L_{p}$ setting, $p \in[1, \infty]$, to see whether they are convex price operators. As we shall see, we have to be cautious with their domains in order to guarantee the Fatou property and then the time-consistency of the whole price system.

This section is divided in three parts. In the first we introduce and study the properties of fully-dynamic risk measures $\left(\rho_{s t}\right)_{s, t}$. Then we define the corresponding risk-indifference price evaluations $\left(x_{s t}\right)_{s, t}$. Here we discuss the very definition of $x_{s t}$ both in terms of domain and range. We shall see that while in the case $p=\infty, x_{s t}$ is a well-defined convex price, for the case $p<\infty$ we have to introduce a new framework beyond the $L_{p}$-setting. It is only in this new framework that we can show that the risk-indifference evaluations $x_{s t}$ have all the properties to be a convex price operator. In particular we obtain the Fatou property and the dual representation of $x_{s t}$. In the last parts of this section we study the time-consistency of the family $\left(x_{s t}\right)_{s, t}$ and the regularity of the trajectories. 


\subsection{Fully-dynamic risk measures, time-consistency, domina- tion, and sensitivity.}

At first we introduce the notion of fully-dynamic risk measure. This object was already considered in [9] under the name of dynamic risk measure.

Definition 2.4. A fully-dynamic convex risk measure $\left(\rho_{s t}\right)_{s, t}$ is a family of convex risk measures indexed by two points in time $s, t \in[0, T]$ such that $s \leq t$. For any $s, t$ with $s \leq t$, the mapping $\rho_{s t}: L_{p}\left(\mathcal{F}_{t}\right) \longrightarrow L_{p}\left(\mathcal{F}_{s}\right)$ satisfies the following properties:

- monotonicity, i.e. $\rho_{s t}\left(X^{\prime}\right) \leq \rho_{s t}\left(X^{\prime \prime}\right)$, for any $X^{\prime} \geq X^{\prime \prime}$ in $L_{p}\left(\mathcal{F}_{t}\right)$,

- convexity, i.e. for any $X^{\prime}, X^{\prime \prime} \in L_{p}\left(\mathcal{F}_{t}\right)$ and $\lambda \in[0,1], \rho_{s t}\left(\lambda X^{\prime}+(1-\right.$ $\left.\lambda) X^{\prime \prime}\right) \leq \lambda \rho_{s t}\left(X^{\prime}\right)+(1-\lambda) \rho_{s t}\left(X^{\prime \prime}\right)$,

- $\mathcal{F}_{s}$-translation invariance, i.e. for any $X \in L_{p}\left(\mathcal{F}_{t}\right), \rho_{s t}(X+f)=$ $\rho_{s t}(X)-f$, for all $f \in L_{p}\left(\mathcal{F}_{s}\right)$.

Moreover,

- If $p=\infty$, we assume that continuity from below holds with convergence point-wise, i.e. for any $X \in L_{\infty}\left(\mathcal{F}_{t}\right)$ and any sequence $\left(X_{n}\right)_{n}$ such that $X_{n} \uparrow X$ P-a.s., then $\rho_{\text {st }}\left(X_{n}\right) \downarrow \rho_{\text {st }}(X) P$-a.s.

We stress that we do not assume a priori that the risk measures $\rho_{s t}$ are normalised.

Remark 2.5. If $p<\infty$, we recall that the risk measure $\rho_{s t}$ is always continuous from below and continuous from above $P$-a.s. (and in $L_{p}$ ). In fact, recall that for any sequence $\left(X_{n}\right)_{n}$ increasing to $X$, the sequence $\left(\rho_{s t}\left(X_{n}\right)\right)_{n}$ is decreasing. Define $\rho(X):=E\left(\rho_{s t}(X)\right), X \in L_{p}\left(\mathcal{F}_{t}\right)$. Then $\rho$ is a static risk measure. Applying the results of [5], [26], we have that $\rho\left(X_{n}\right) \downarrow \rho(X), n \rightarrow \infty$. The above mentioned monotonicity of $\rho_{\text {st }}$ implies that $\rho_{s t}\left(X_{n}\right) \downarrow \rho_{s t}(X) P$-a.s., which in turns implies also the $L_{p}$-convergence.

Remark 2.6. The risk measure $\rho_{s t}$ satisfies weak $\mathcal{F}_{s^{-}}$-homogeneity, i.e.

$$
1_{A} \rho_{s t}(X)=1_{A} \rho_{s t}\left(1_{A} X\right), \quad X \in L_{p}\left(\mathcal{F}_{t}\right), \quad A \in \mathcal{F}_{s} .
$$

Indeed, for $p=\infty$, monotonicity and translation invariance imply (2.4) for all $X \in L_{\infty}\left(\mathcal{F}_{t}\right)$. See [18] Proposition 3.3 and [33] Section 3. In the case $p<\infty$, the weak $\mathcal{F}_{s}$-homogeneity for all $X \in L_{p}\left(\mathcal{F}_{t}\right)$ is then guaranteed by the continuity from below.

Remark 2.7. The risk measures $\rho_{\text {st }}$ satisfy a stronger convexity property, i.e. for any $X, Y \in L_{p}\left(\mathcal{F}_{t}\right)$, it is

$$
\rho_{s t}(\Lambda X+(1-\Lambda) Y) \leq \Lambda \rho_{s t}(X)+(1-\Lambda) \rho_{s t}(Y),
$$

for $\Lambda \in L_{\infty}\left(\mathcal{F}_{s}\right)$ with $0 \leq \Lambda \leq 1$. The property follows from [33] Remark 2, page 602. 
Proposition 2.8. For any $s \leq t$ the risk measure above admits the following representation:

$$
\begin{aligned}
\rho_{s t}(X)=\underset{\substack{Q \ll P: \\
Q_{\mid \mathcal{F}_{s}}=P_{\mid \mathcal{F}_{s}}}}{\operatorname{essmax}}\left(E_{Q}\left[-X \mid \mathcal{F}_{s}\right]-\alpha_{s t}(Q)\right) \\
=\underset{\substack{Q \ll P: \\
E_{P}\left[\alpha_{s t}(Q)\right]<\infty}}{\operatorname{essmax}}\left(E_{Q}\left[-X \mid \mathcal{F}_{s}\right]-\alpha_{s t}(Q)\right), \quad X \in L_{p}\left(\mathcal{F}_{t}\right),
\end{aligned}
$$

where $\alpha_{s t}$ is the minimal penalty, i.e.

$$
\alpha_{s t}(Q):=\operatorname{esssup}_{X \in L_{p}\left(\mathcal{F}_{t}\right)}\left(E_{Q}\left[-X \mid \mathcal{F}_{s}\right]-\rho_{s t}(X)\right) .
$$

Proof. The proof is given in [8] in the case $p=\infty$. Here we propose a self-contained proof inspired by [22]. The inequality

$$
\rho_{s t}(X) \geq \operatorname{esssup}_{\substack{Q \ll P: \\ \mathcal{F}_{\mid \mathcal{F}_{s}}=P_{\mid \mathcal{F}_{s}}}}\left(E_{Q}\left[-X \mid \mathcal{F}_{s}\right]-\alpha_{s t}(Q)\right)
$$

follows from the definition of the minimal penalty. Define $\rho(X):=E\left(\rho_{s, t}(X)\right)$, $X \in L_{p}\left(\mathcal{F}_{t}\right)$. Then $\rho$ is a static risk measure, continuous from below and it admits representation

$$
\rho(X)=\max _{Q \ll P}\left(E_{Q}(-X)-\alpha(Q)\right),
$$

where $\alpha(Q)$ is the minimal penalty for $\rho$. See [28] in the case $p=\infty$ and see [26] for $p<\infty$. As in the proof of the dual representation in the case of continuity from above (cf. [22] or [1]), $\alpha(Q)<\infty$ implies that $Q \ll P$, $Q_{\mid \mathcal{F}_{s}}=P_{\mid \mathcal{F}_{s}}$ and $E\left(\alpha_{s t}(Q)\right) \leq \alpha(Q)$.

For any $X$, let $Q_{X}$ be an $\operatorname{argmax}$ in (2.7). Then we have

$$
\begin{aligned}
\rho(X) & =E_{Q_{X}}(-X)-\alpha\left(Q_{X}\right) \\
& \leq E\left[E_{Q_{X}}\left(-X \mid \mathcal{F}_{s}\right)-\alpha_{s t}\left(Q_{X}\right)\right] \\
& \leq E\left(\rho_{s t}(X)\right)=\rho(X)
\end{aligned}
$$

This proves that $E_{Q_{X}}\left(-X \mid \mathcal{F}_{s}\right)-\alpha_{s t}\left(Q_{X}\right)=\rho_{s t}(X)$.

Lemma 2.9. Given representation (2.5), let $Q$ be a probability measure such that $E\left(\alpha_{s t}(Q)\right)<\infty$. Then we have

$$
\alpha_{s t}(Q)=\operatorname{esssup}_{X \in L_{\infty}\left(\mathcal{F}_{t}\right)}\left(E_{Q}\left(-X \mid \mathcal{F}_{s}\right)-\rho_{s t}(X)\right) .
$$

Proof. We only need to prove (2.8) in the case where $p<\infty$. It is well known that $\alpha_{s t}(Q)=\operatorname{esssup}_{X \in \mathcal{A}_{s t}^{p}} E_{Q}\left(-X \mid \mathcal{F}_{s}\right)$ where $\mathcal{A}_{s t}^{p}$ is the acceptance 
set for $\rho_{s t}$, i.e. $\mathcal{A}_{s t}^{p}:=\left\{X \in L_{p}\left(\mathcal{F}_{t}\right) \mid \rho_{s t}(X) \leq 0\right\}$. See [22]. Let $X \in$ $\mathcal{A}_{s t}^{p}$. For all $n>0$, let $X_{n}:=\sup (X,-n)$. Every $X_{n}$ belongs to $\mathcal{A}_{s t}^{p} \subseteq$ $L_{p}\left(\mathcal{F}_{t}\right)$. The sequence $\left(-X_{n}\right)_{n}$ is increasing to $-X$. The monotone convergence theorem shows that $E_{Q}\left(-X_{n} \mid \mathcal{F}_{s}\right)$ is increasing to $E_{Q}\left(-X \mid \mathcal{F}_{s}\right)$. Then the continuity from above of $\rho_{s t}$ allows to restrict the evaluation of $\operatorname{esssup}_{X \in L_{p}\left(\mathcal{F}_{t}\right)}\left[E_{Q}\left(-X \mid \mathcal{F}_{s}\right)-\rho_{s t}(X)\right]$ to the elements $X$ bounded from below. So, let $X \in L_{p}\left(\mathcal{F}_{t}\right)$ be bounded from below by $C$. Then $X$ is the increasing limit of the sequence of bounded random variables $Y_{n}:=\inf (X, n)$. Moreover, $\left|Y_{n}\right| \leq \sup (|C|, X)$. The hypothesis $E\left(\alpha_{s t}(Q)\right)<\infty$ implies that $Q \ll P$ with a density in $L_{q}\left(\mathcal{F}_{t}\right)$, where $q$ is the conjugate of $p$. We obtain that $E_{Q}\left(-X \mid \mathcal{F}_{s}\right)=\lim _{n \rightarrow \infty} E_{Q}\left(-Y_{n} \mid \mathcal{F}_{s}\right)$, from the dominated convergence theorem for conditional expectations. On the other hand, $\rho_{s t}$ is continuous from below, thus $\rho_{s t}(X)=\lim _{n \rightarrow \infty} \rho_{s t}\left(Y_{n}\right)$. This proves that

$$
E_{Q}\left(-X \mid \mathcal{F}_{s}\right)-\rho_{s t}(X) \leq \operatorname{esssup}_{X \in L_{\infty}\left(\mathcal{F}_{t}\right)}\left[E_{Q}\left(-X \mid \mathcal{F}_{s}\right)-\rho_{s t}(X)\right]
$$

The result follows easily.

\subsubsection{About time-consistency.}

We give two notions of time-consistency for fully-dynamic risk measures.

Definition 2.10. A fully-dynamic risk measure on $L_{p}$ is

- strong time-consistent if for all $r, s, t \in[0, T]: r \leq s \leq t$, we have

$$
\rho_{r t}(X)=\rho_{r s}\left(-\rho_{s t}(X)\right), \quad X \in L_{p}\left(\mathcal{F}_{t}\right),
$$

- time-consistent if for all $r, s, t \in[0, T]: r \leq s \leq t$, for all $X, Y \in$ $L_{p}\left(\mathcal{F}_{t}\right)$, we have

$$
\rho_{s t}(X)=\rho_{s t}(Y) \quad \Longrightarrow \quad \rho_{r t}(X)=\rho_{r t}(Y) .
$$

The second concept, here called simply time-consistency, derives directly from the same notion introduced for classical dynamic risk-measures $\left(\rho_{s}\right)_{s}$, indexed by one time parameter, see [1] and see e.g. [6] for a recent survey on various weaker concepts of time-consistency.

\section{Remark 2.11.}

1. Time consistency is substantially an "order preserving property". Indeed, from monotonicity and translation invariance, we have that time consistency as defined above is equivalent to the order preserving property: For all $r \leq s \leq t, \rho_{s t}(X) \geq \rho_{s t}(Y)$, implies $\rho_{r t}(X) \geq \rho_{r t}(Y)$. 
2. It is easy to see that time-consistency is transferred to the normalised version

$$
\check{\rho}_{s t}(X):=\rho_{s t}(X)-\rho_{s t}(0), \quad X \in L_{p}\left(\mathcal{F}_{t}\right),
$$

of $\left(\rho_{s t}\right)_{s, t}$ and, more generally, to any translation of $\left(\rho_{s t}\right)_{s, t}$.

The concept of strong time-consistency, where strong is used to distinguish it from the other one, was already introduced in [9], where a complete characterisation was also provided.

\section{Remark 2.12.}

1. Strong time-consistency is a "composition rule". It means that for all $r \leq s \leq t$, the risk at time $r$ associated to any $\mathcal{F}_{t}$-measurable random variable $X$ can be computed either directly as $\rho_{r t}(X)$ or making use of the instant of time $s$ as $\rho_{r s}\left(-\rho_{s t}(X)\right)$, and both quantities are the same. We recall that strong time-consistency is fully characterised in terms of the cocycle condition for the minimal penalties. See Theorem 1 in [10].

2. The property of strong time-consistency is not transferred to the normalised version $\left(\check{\rho}_{s t}\right)_{s, t}$ of $\left(\rho_{s t}\right)_{s, t}$. To see this, assume that the fullydynamic risk measure $\left(\rho_{s t}\right)_{s, t}$ satisfies strong time-consistency, which is equivalent to the cocycle condition. From the definition of minimal penalty (2.6), we have that the associated normalised fully-dynamic risk measure $\left(\check{\rho}_{\text {st }}\right)$ satisfies also strong time-consistency if and only if, for all probability measure $Q$ such that $E_{Q}\left(\alpha_{r t}(Q)\right)<\infty, \rho_{r t}(0)=$ $\rho_{r s}(0)+E_{Q}\left(\rho_{s t}(0) \mid \mathcal{F}_{r}\right)$. This condition is, in fact, very strong.

The following result details the relationship between the two forms of timeconsistency. One can also compare this with the concepts of time-consistency for price operators (see Definition 2.2).

Proposition 2.13. Let $\rho_{s t}: L_{p}\left(\mathcal{F}_{t}\right) \longrightarrow L_{p}\left(\mathcal{F}_{s}\right), s, t \in[0, T]: s \leq t$. The following assertions are equivalent:

i) The fully-dynamic risk measure $\left(\rho_{s t}\right)_{s, t}$ is strong time-consistent.

ii) The fully-dynamic risk measure $\left(\rho_{s t}\right)_{s, t}$ is time-consistent and

$$
\rho_{r t}(Y)=\rho_{r s}\left(Y-\rho_{s t}(0)\right), \quad 0 \leq r \leq s \leq t, \quad Y \in L_{p}\left(\mathcal{F}_{s}\right) .
$$

Proof. To see that (i) implies (ii), it is enough to use the translation invariance property of $\rho_{s t}$. Conversely, assume that (ii) is satisfied. Let $Z \in L_{p}\left(\mathcal{F}_{t}\right)$ and define $Y:=\rho_{s t}(0)-\rho_{s t}(Z)$. From the translation invariance property of $\rho_{s t}$ we have that $\rho_{s t}(Y)=\rho_{s t}(0)-\left[\rho_{s t}(0)-\rho_{s t}(Z)\right]=\rho_{s t}(Z)$. Thus, from time-consistency, $\rho_{r t}(Y)=\rho_{r t}(Z)$. Applying (2.9) to $Y$, we get that $\rho_{r t}(Z)=\rho_{r s}\left(-\rho_{s t}(Z)\right)$. 
When the risk measures $\left(\rho_{s t}\right)_{s, t}$ are normalised, the above result has an easy interpretation.

Corollary 2.14. Assume that the fully-dynamic risk measure $\left(\rho_{s t}\right)_{s, t}$ is normalised, i.e. $\rho_{s t}(0)=0$ for all $s \leq t$. Then the fully-dynamic risk measure $\left(\rho_{s t}\right)_{s, t}$ is strong time-consistent if and only if it is time-consistent and satisfies the restriction property, i.e. for all $0 \leq r \leq s \leq t$,

$$
\rho_{r t}(Y)=\rho_{r s}(Y), \quad Y \in L_{p}\left(\mathcal{F}_{s}\right) .
$$

To summarise, in this work we consider a strong time-consistent fullydynamic risk measure $\left(\rho_{s t}\right)_{s, t}$ not necessarily normalised. Also we do not assume the restriction property (2.10).

\subsubsection{About domination.}

The concept of domination for a risk measure will be crucial for defining the correct framework to study risk-indifference price operators.

Definition 2.15. Fix $p<\infty$. Let $\rho: L_{p}\left(\mathcal{F}_{T}\right) \longrightarrow \mathbb{R}$ be a convex risk measure continuous from below. The risk measure $\rho$ is dominated if there exists a sublinear (or coherent) risk measure $\tilde{\rho}: L_{p}\left(\mathcal{F}_{T}\right) \rightarrow \mathbb{R}$ such that

$$
\rho(X)-\rho(0) \leq \tilde{\rho}(X), \quad X \in L_{p}\left(\mathcal{F}_{T}\right) .
$$

The property of domination was first introduced in [15], where it is also proved that it guarantees a representation result. The following proposition summarises these findings.

Proposition 2.16. Fix $p \in[1, \infty)$. Let $\rho: L_{p}\left(\mathcal{F}_{T}\right) \longrightarrow \mathbb{R}$ be a convex risk measure. The following statements are equivalent:

1. The risk measure $\rho$ is dominated.

2. There exist $K>0$ and $C \in \mathbb{R}$ such that $\rho(X) \leq K\|X\|_{p}+C$, for all $X \in L_{p}\left(\mathcal{F}_{T}\right)$

3. The risk measure $\rho$ admits representation

$$
\rho(X)=\max _{Q \in \mathcal{B}^{K}}\left(E_{Q}(-X)-\alpha(Q)\right), \quad X \in L_{p}\left(\mathcal{F}_{T}\right),
$$

where $\mathcal{B}^{K}$ is the set of probability measures on $\mathcal{F}_{T}$ :

$$
\mathcal{B}^{K}:=\left\{Q \ll P: \frac{d Q}{d P} \in L_{q}\left(\mathcal{F}_{T}\right),\left\|\frac{d Q}{d P}\right\|_{q} \leq K\right\}, \quad q=p(p-1)^{-1},
$$

and $\alpha$ is the minimal penalty for $\rho$. 
Proof. Whenever $\Omega$ is a Polish space, Proposition 3.1 [15] apply directly to $L_{p}(\Omega, \mathcal{B}(\Omega), P)$ (with $\left.c(f)=E\left(|f|^{p}\right)^{1 / p}\right)$. However, that result is also valid for $L_{p}(\Omega, \mathcal{F}, P)$ without any assumption on $(\Omega, \mathcal{F}, P)$. Indeed the representation

$$
\rho(X)=\max _{Q \in L_{p}(\mathcal{F})^{*}}\left(E_{Q}(-X)-\alpha(Q)\right)
$$

follows from the extended Namioka-Klee Theorem ([5] Theorem 1). Replacing $L^{1}(c)$ by $L_{p}(\Omega)$ and $c(f)$ by $\|f\|_{p}$ everywhere in the proof of Proposition $3.1[15]$, we obtain the result for general $L_{p}\left(\mathcal{F}_{T}\right)$.

The above representation is known in the case of sublinear (or coherent) risk measures, see [26] and [31]. Also a representation result for finite convex risk measures on $L_{p}(p<\infty)$ is given in [31] Theorem 2.11. This proof is unfortunately based on a wrong statement (see Proposition 2.10 in [31]).

Note that, for a dominated convex risk measure $\rho$, we have the sandwich:

$$
-\tilde{\rho}(-X) \leq \rho(X)-\rho(0) \leq \tilde{\rho}(X), \quad X \in L_{p}\left(\mathcal{F}_{T}\right),
$$

where $-\tilde{\rho}(-X)$ is superlinear and $\tilde{\rho}$ is sublinear. The sandwich relationship above provides a motivation in itself for the use of domination. This is in view of the extension theorems of strong time-consistent convex operators satisfying a sandwich condition as studied in [14]. Indeed if we consider a strong time-consistent family of operators $\left(\rho_{s t}\right)_{s, t}$ defined on the vector subspaces $\left(L_{t}\right)_{t}$ with $L_{t} \subseteq L_{p}\left(\mathcal{F}_{t}\right)$ and if each $\rho_{s t}$ satisfies a sandwich condition and the Fatou property, then we can extend this family $\left(\rho_{s t}\right)_{s, t}$ to the whole $\left(L_{p}\left(\mathcal{F}_{t}\right)\right)_{t}$.

\subsubsection{About sensitivity.}

The concept of sensitivity for a risk measure yields a representation of the very risk measure in terms of probability measures equivalent to $P$. See e.g. Section 3 in [33].

Definition 2.17. Let $\rho: L_{p}\left(\mathcal{F}_{T}\right) \longrightarrow \mathbb{R}$ be a convex risk measure.

- The risk measure $\rho$ is strong sensitive or relevant (to $P$ ), if

$$
\rho\left(1_{B}\right)<\rho(0),
$$

for all $B \in \mathcal{F}_{T}$ such that $P(B)>0$.

- The risk measure $\rho$ is sensitive (to $P$ ) if there exists a probability measure $\tilde{Q} \sim P$ such that $\alpha(\tilde{Q})<\infty$, where $\alpha$ is the minimal penalty associated to $\rho$.

Remark 2.18. The property of relevance implies sensitivity. This follows from Lemma 3.4 in [33] applied to $\Phi(X)=-\rho(X)$, restricted to $L_{\infty}\left(\mathcal{F}_{T}\right)$. 
Proposition 2.19. Fix $p<\infty$. Let $\rho: L_{p}\left(\mathcal{F}_{T}\right) \longrightarrow \mathbb{R}$ be a sensitive convex risk measure, then the risk measure $\rho$ admits representation

$$
\rho(X)=\sup _{Q \in \mathcal{Q}}\left(E_{Q}(X)-\alpha(Q)\right), \quad X \in L_{p}\left(\mathcal{F}_{T}\right),
$$

where

$$
\mathcal{Q}:=\{Q \sim P: \quad \alpha(Q)<\infty\} .
$$

Moreover, if $\rho$ is dominated, then $\mathcal{Q} \subseteq \mathcal{B}^{K}$, where $K$ is the constant in the domination property and $q=p(p-1)^{-1}$.

Proof. Representation (2.12) is a direct consequence of Theorem 3.1 in [33]. Let $Q \in \mathcal{Q}$, then the domination implies that $E_{Q}(X) \leq \alpha(Q)+K\|X\|_{p}+C$. Applying this to $\lambda X$ for all $\lambda>0$ we have

$$
E_{Q}[X] \leq \frac{\alpha(Q)+C}{\lambda}+K\|X\|_{p}
$$

By taking $\lambda \rightarrow \infty$ we conclude.

\subsubsection{Fully-dynamic risk measures vs. dynamic risk-measure}

In large part of the literature on risk-measures, a so-called dynamic risk measure $\left(\rho_{s}\right)_{s}$ is characterised by only one time index. See e.g. [1], [33]. In this case $\rho_{s}$ represents to the evaluation criterion of any position at a fixed time horizon $T<\infty$ and this corresponds to $\rho_{s}:=\rho_{s T}$, in terms of fully-dynamic risk measures $\left(\rho_{s t}\right)_{s, t}$.

In particular, with the mere use of dynamic risk measures, we have that the risk evaluation of any $X \in L_{p}\left(\mathcal{F}_{t}\right)$ is the same for every $t \in[s, T]$. In the framework of fully-dynamic risk measures this situation corresponds to the restriction property $(2.10)$ for $\left(\rho_{s t}\right)_{s, t}$ :

$$
\rho_{s}(X)=\rho_{s T}(X)=\rho_{s t}(X) \quad X \in L_{p}\left(\mathcal{F}_{t}\right), \quad s \leq t \leq T .
$$

Moreover, recall that time-consistency for a dynamic risk measure $\left(\rho_{t}\right)_{t}$ is

$$
\rho_{t}(X)=\rho_{t}(Y) \quad \Longrightarrow \quad \rho_{s}(X)=\rho_{s}(Y), \quad s \leq t, \quad X, Y \in L_{p}\left(\mathcal{F}_{T}\right) .
$$

From Corollary 2.14, we can see that if the fully-dynamic risk measure $\left(\rho_{s t}\right)_{s, t}$ is normalised, i.e. $\rho_{s t}(0)=0$ for all $s \leq t$, then there is a one-to-one correspondence between the strong time-consistent $\left(\rho_{s t}\right)_{s, t}$ and the timeconsistent $\left(\rho_{s}\right)_{s}$. In fact,

$$
\rho_{s t}(X)=\rho_{s T}(X)=\rho_{s}(X), \quad X \in L_{p}\left(\mathcal{F}_{t}\right), \quad t \geq s .
$$

We stress that the result is not true if $\left(\rho_{s t}\right)_{s, t}$ is not normalised. 
Remark 2.20. The idea of having an evaluation criteria evolving with time is also identified in [37], where the concept of dynamic performance is detailed together with the one of forward performance, i.e. a dynamic performance with a specification at the initial time. These are discussed in the context of portfolio optimisation and indifference prices in markets with explicitly modelled price dynamics, e.g. the binomial model in [36] and an Itô type dynamic in [37]. The dynamic performance is intended to extend the concept of value process generated (backwards) by a utility function typically by dynamic programming. The dynamic performance $\left(U_{t}(x)\right)_{t}$ is in fact only capturing those essential properties that such value process possess: the adaptedness to a given information flow, the supermartingality of $\left(U_{t}(X)\right)_{t}$ for any attainable $X$, and the martingality of $\left(U_{t}\left(X^{*}\right)\right)_{t}$, for an optimal and attainable $X^{*}$. We remark that the dynamic performance $\left(U_{t}(x)\right)_{t}$ is characterised by one time index only. Also, in general, for $s<t$ and an $\mathcal{F}_{s}$-measurable $X, U_{s}(X) \neq U_{t}(X)$. In this they differ from dynamic risk-measures as fully-dynamic risk measures do (i.e. there is no à priori restriction property (2.10)). On the other side, a dynamic convex risk measures plus its minimal penalty $V_{t}(X):=\rho_{t}(X)+\alpha_{t}(Q), t \in[0, T]$, is a $Q$-supermartingale, for $X$ fixed, and it is a $Q$-martingale if the maximum is achieved at $Q$ in the robust representation of $\rho_{0}(X)$. See [1].

\subsubsection{Example: Strong time-consistent fully-dynamic risk mea- sures from BMO martingales}

Examples of strong time-consistent fully-dynamic risk measures on $L_{\infty}$ were constructed in [9] making use of BMO martingales, cf. Proposition 4.13 and Proposition 4.19. Here we present the $L_{p}, p<\infty$, case. Recall the following definition of BMO martingales.

Definition 2.21. A right continuous uniformly integrable martingale $M$ is $B M O$ if there is a constant $C$ such that for any stopping time $S$,

$$
E\left([M, M]_{\infty}-[M, M]_{S^{-}} \mid \mathcal{F}_{S}\right) \leq C^{2} .
$$

The smallest $C$ is, by definition, the BMO norm of $M:\|M\|_{B M O}$.

For the study of right-continuous BMO martingales, we refer to [24] and for continuous BMO martingales we refer to [32]. In the case of continuous martingales, the above norm $\|M\|_{B M O}$ is often written $\|M\|_{B M O_{2}(P)}$. We prove now that the construction detailed in [9] gives also rise to strong time-consistent fully-dynamic risk measures on $L_{p}$ spaces when the set of martingales used for the construction of the dynamic risk measure is a set of BMO martingales uniformly bounded. Note that for given $K>0$, the set

$$
\mathcal{M}_{K}=\left\{M: B M O \text { martingale, }\|M\|_{B M O} \leq K\right\}
$$


is a stable set. We first prove the following lemma for conditional expectations with respect to the probability measures $Q_{M} \ll P$ with RadonNikodym derivative

$$
\frac{d Q_{M}}{d P}=\mathcal{E}(M)_{T} \quad \text { on } \mathcal{F}_{T}
$$

associated to some continuous BMO martingale $M$.

Lemma 2.22. Let $K>0$. There is $p(K)>0$, depending only on $K$, such that for all continuous BMO martingale $M$ with $\|M\|_{B M O} \leq K$, the conditional expectation $E_{Q_{M}}\left(. \mid \mathcal{F}_{s}\right)$ defines a continuous operator:

$$
E_{Q_{M}}\left(. \mid \mathcal{F}_{s}\right): L_{p}\left(\mathcal{F}_{T}\right) \longrightarrow L_{p}\left(\mathcal{F}_{s}\right), \quad p>p(K) .
$$

Furthermore, the norm of this operator is bounded by some constant depending only on $K$ and $p$.

Proof. From Theorem 3.1 of [32], there exists $q(K) \in(1, \infty)$, such that for $q<q(K)$, for all $M$ with $\|M\|_{B M O} \leq K$, the stochastic exponential $\mathcal{E}(M)$ satisfies the reverse Hölder inequality:

$$
E\left(\mathcal{E}(M)_{t}^{q} \mid \mathcal{F}_{s}\right) \leq C_{q} \mathcal{E}(M)_{s}^{q}
$$

for some constant $C_{q}$ depending only on $K$ and $q$.

Let $p>p(K)=\frac{q(K)}{q(K)-1}$. Thanks to the Hölder inequality for conditional expectations and (2.13), we have that that, for all $X \in L_{p}\left(\mathcal{F}_{T}\right)$,

$$
\left|E_{Q_{M}}\left(X \mid \mathcal{F}_{s}\right)\right| \leq\left[\left(E\left(\frac{\mathcal{E}(M)_{T}}{\mathcal{E}(M)_{s}}\right)^{q} \mid \mathcal{F}_{s}\right)\right]^{\frac{1}{q}} E\left(|X|^{p} \mid \mathcal{F}_{s}\right)^{\frac{1}{p}} \leq C_{q}^{\frac{1}{q}} E\left(|X|^{p} \mid \mathcal{F}_{s}\right)^{\frac{1}{p}} .
$$

From this we have $E\left(\left|E_{Q_{M}}\left(X \mid \mathcal{F}_{s}\right)\right|^{p}\right)^{\frac{1}{p}} \leq C_{q}^{\frac{1}{q}}\|X\|_{p}$.

We can now construct a fully-dynamic risk measure on $L_{p}$.

Proposition 2.23. Let $\mathcal{M}$ be a stable set of BMO continuous martingales with BMO norm bounded by $K$. Let $b_{u}, u \in[0, T]$, be a bounded predictable process. For all $0 \leq s \leq t \leq T$, let

$$
\rho_{s t}(X)=\operatorname{esssup}_{M \in \mathcal{M}}\left(E_{Q_{M}}\left(-X \mid \mathcal{F}_{s}\right)-\alpha_{s t}\left(Q_{M}\right)\right)
$$

where $Q_{M}$ is as above, and

$$
\alpha_{s t}\left(Q_{M}\right)=E_{Q_{M}}\left(\int_{s}^{t} b_{u} d[M, M]_{u} \mid \mathcal{F}_{s}\right) .
$$

Then $\left(\rho_{s t}\right)_{s, t}$ defines a fully-dynamic risk measure on $\left(L_{p}\left(\mathcal{F}_{t}\right)\right)_{t}$ for every $p(K)<p<\infty$, with $p(K) \in(1, \infty)$ depending only on $K$. Moreover, $\left(\rho_{s t}\right)_{s, t}$ is strong time-consistent. Also, $\rho_{0 T}$ is dominated and sensitive. 
Proof. It is proved in the proof of Proposition 4.13 of [9] that, for all $M$,

$$
\left\|\alpha_{s t}\left(Q_{M}\right)\right\|_{\infty} \leq\left(\sup _{u}\left\|b_{u}\right\|_{\infty}\right)\|M\|_{B M O_{2}\left(Q_{M}\right)}
$$

From Lemma 4.12 of [10] and the assumption on $\mathcal{M}$, we have that

$$
\sup _{M \in \mathcal{M}}\left\|\alpha_{s t}\left(Q_{M}\right)\right\|_{\infty}<\infty
$$

From Lemma 2.22, we obtain that, for all $X \in L_{p}\left(\mathcal{F}_{t}\right)$,

$$
\left\|\rho_{s t}(X)\right\|_{p} \leq C_{q}^{\frac{1}{q}}\|X\|_{p}+C,
$$

for some $C>0$. This proves that $\rho_{s t}$ is well-defined on $L_{p}\left(\mathcal{F}_{t}\right)$ with values on $L_{p}\left(\mathcal{F}_{s}\right)$. Furthermore, the above equation proves that $\rho_{0 T}$ is dominated. The sensitivity of $\rho_{0 T}$ follows from (2.15) and the observation that every $Q_{M}$ is equivalent to $P$ on $\mathcal{F}_{T}$. It is also proved in [9] that the penalties $\alpha_{s t}\left(Q_{M}\right)$ satisfy the local condition and the cocycle condition. Strong timeconsistency follows then from [10].

\section{Remark 2.24.}

1. Making use of the arguments presented in [9] Section 4.4, the above proof can be adapted to the case of BMO martingales with jumps, considering right-continuous BMO martingales with BMO norm uniformly bounded by $K<\frac{1}{16}$.

2. Other examples of strong time-consistent fully-dynamic risk measures defined on $L_{p} 1 \leq p \leq \infty$ are constructed in Theorem 3 of [12] making use of probability measures solutions to path-dependent martingale problems. These dynamic risk measures are linked to solutions to Path Dependent PDEs.

\subsubsection{Example: Strong time-consistent fully dynamic risk mea- sures from BSDEs}

In this example we consider a $d$-dimensional Brownian motion $W$ on $(\Omega, \mathcal{F}, P)$ and the corresponding $P$-completed filtration $\left(\mathcal{F}_{t}\right)_{t}$ generated by $W$. We consider the following BSDE

$$
Y_{s}=-\xi+\int_{s}^{t} g\left(u, Z_{u}\right) d u-\int_{s}^{t} Z_{u} d W_{u}
$$

where $0 \leq s \leq t \leq T, \xi$ is a $\mathcal{F}_{t}$-measurable terminal condition and $g(u, \omega, z)$, $u \in[0, T], \omega \in \Omega, z \in \mathbb{R}^{d}$, is a $\mathcal{F}_{t}$-adapted driver. 
Proposition 2.25. Assume that $g$ is $\mathbb{R}$-valued, convex in $z$, and uniformly Lipschitz with respect to $z$, i.e. there exists $C>0$,

$$
\left|g(u, z)-g\left(u, z^{\prime}\right)\right| \leq C|| z-z^{\prime} \|
$$

and $E\left(\int_{0}^{T}|g(u, 0)|^{2} d u\right)<\infty$. Let $Y_{s}$ be given by the unique solution to the $B S D E$ (2.16). Then $\rho_{s t}(\xi):=Y_{s}$ defines a fully-dynamic risk measure on $\left(L_{2}\left(\mathcal{F}_{t}\right)\right)_{t}$. This fully-dynamic risk measure is strong time-consistent.

Proof. Pardoux and Peng proved in [41] the existence and uniqueness of a solution $\left(Y_{s}, Z_{s}\right)_{0 \leq s \leq t}$ to $(2.16)$ such that $E\left(\sup _{0 \leq s \leq t}\left|Y_{s}\right|^{2}\right)<\infty$ and $E\left(\int_{0}^{T}\left\|Z_{u}\right\|^{2} d u\right)<\infty$ for all $\xi \in L_{2}\left(\mathcal{F}_{t}\right)$. Proposition 6.7 of [3] yields that $\left(\rho_{s t}\right)_{s, t}$ defines a strong time-consistent fully-dynamic risk measure on $\left(L_{2}\left(\mathcal{F}_{t}\right)\right)_{t}$.

Proposition 2.26. Assume that $g$ is $\mathbb{R}$-valued, convex, continuous in $z$ with quadratic growth, i.e. there exists $k>0$ such that $(t, \omega)$ a.e. $|g(u, \omega, z)| \leq$ $k\left(1+\|z\|^{2}\right)$. Furthermore, assume that $g$ is differentiable in $z$ and that there exists $c>0$ such that $\frac{\partial g}{\partial z} \leq c(1+\|z\|)$. Let $Y_{s}$ be given by the unique maximal solution to the BSDE (2.16), Then $\rho_{s t}(\xi):=Y_{s}$ defines a fully-dynamic risk measure on $\left(L_{\infty}\left(\mathcal{F}_{t}\right)\right)_{t}$. This fully-dynamic risk measure is strong time-consistent.

Proof. Kobylanski proved in [34] the existence and uniqueness of a maximal bounded solution to $(2.16)$ for all $\xi \in L_{\infty}\left(\mathcal{F}_{t}\right)$. From Proposition 6.7 of [3], we have that $\left(\rho_{s t}\right)_{s, t}$ defines a strong time-consistent fully-dynamic risk measure on $\left(L_{\infty}\left(\mathcal{F}_{t}\right)\right)_{t}$.

Observe that the risk measures constructed here above are not normalised, in general. In fact we have the following result.

Corollary 2.27. If in Proposition 2.25 and in Proposition 2.26, we assume in addition that $g(t, \omega, 0)=0(t, \omega)$ - a.e., then the corresponding strong time-consistent fully-dynamic risk measures $\left(\rho_{s t}\right)_{s, t}$ are normalised and thus satisfy the restriction property (2.10). Thus $\left(\rho_{s t}\right)_{s, t}$ defines a conditional g-expectation as introduced by Peng [42].

Remark 2.28. Under the assumptions of Proposition 2.26 (quadratic growth in $z), \rho_{s t}$ admits a dual representation of the kind (2.14) (cf [3]) with a set of BMO continuous martingales. However, in general the BMO norms of these BMO martingales are not uniformly bounded. Therefore in general it is not possible to find $p<\infty$ such that $\left(\rho_{s t}\right)_{s, t}$ defines a fully-dynamic risk measure on $\left(L_{p}\left(\mathcal{F}_{t}\right)\right)_{t}$.

Remark 2.29. We also recall the following result from [20]. On the given $P$-completed Brownian filtration, every normalised strong time-consistent 
fully-dynamic risk measure $\left(\rho_{s t}\right)_{s, t}$ is the increasing limit of a sequence of normalised strong time-consistent fully-dynamic risk measures $\left(\rho_{s t}^{n}\right)_{s, t}$, where each $\rho_{s t}^{n}$ is associated to a BSDE with a convex Lipschitz driver $g_{n}$.

\subsection{A risk-indifference price operator $x_{s t}$.}

Risk-indifferent pricing was introduced in a static set-up as an alternative pricing technique to utility-indifference pricing in incomplete markets. Instead of considering the agents' attitude to a financial investment in terms of utility functions, the risk-indifference approach uses risk measures. The connection between these two approaches is given by the fact that utilityindifference with exponential utility function corresponds substantially to a risk-indifference pricing with entropic risk measure.

Hereafter we consider the whole family of risk-indifference prices $\left(x_{s t}\right)_{s, t}$ generated by a strong time-consistent fully-dynamic risk measure $\left(\rho_{s t}\right)_{s, t}$ on $\left(L_{p}\left(\mathcal{F}_{t}\right)\right)_{t}, 1 \leq p \leq \infty$. Risk-indifference pricing is also studied in [33] for the case of dynamic risk measures $\left(\rho_{t}\right)_{t}$ as in Subsection 2.1.4 and in the case $p=\infty$ only. Our first goal is to identify the conditions under which a risk-indifferent price evaluation $x_{s t}$, associated to $\rho_{s t}$, satisfies the properties for being a convex price operator.

First of all fix $s, t \in[0, T]: s \leq t$. From a qualitative perspective, the riskindifference (seller's discounted) price $x_{s t}(X)$ at time $s$ for any (discounted) financial position $X$, at time $t$, is given by the equation:

$$
\underset{\theta \in \Theta_{s t}}{\operatorname{essinf}} \rho_{s t}\left(x_{s t}(X)+y_{s}+Y_{s t}(\theta)-X\right)=\underset{\theta \in \Theta_{s t}}{\operatorname{essinf}} \rho_{s t}\left(y_{s}+Y_{s t}(\theta)\right) \quad P-a . s .,
$$

where $y_{s} \in L_{p}\left(\mathcal{F}_{s}\right)$ represents the money market account and, together with the price $x_{s t}(X)$, is the initial capital at $s$. The $\mathcal{F}_{t}$-measurable $Y_{s t}(\theta)$ represents the value of an admissible portfolio $\theta$ on the time horizon $(s, t]$ and the set $\Theta_{s t}$ represents the admissible portfolios. Under suitable integrability conditions, the equation above can be rewritten as

$$
x_{s t}(X)=\underset{\theta \in \Theta_{s t}}{\operatorname{essinf}} \rho_{s t}\left(Y_{s t}(\theta)-X\right)-\underset{\theta \in \Theta_{s t}}{\operatorname{essinf}} \rho_{s t}\left(Y_{s t}(\theta)\right)
$$

by the translation invariance of $\rho_{s t}$. Here below we give details about portfolios and value processes, so to achieve a general definition of riskindifference price. Equation (2.18) makes sense for all those strategies for which $Y_{s t}(\theta) \in L_{p}\left(\mathcal{F}_{t}\right)$, for all $t$. In this paper we aim at a definition of riskindifference price applicable with large generality. Then we proceed with the following set of definitions.

The market is characterised by a number of underlying assets, whose (discounted) price is given by $\left(\Pi_{t}\right)_{t \in[0, T]}$ which is a $\mathbb{F}$-adapted locally bounded semimartingale in $\mathbb{R}^{d}$. 
Definition 2.30. Denote $\Xi$ the set of all $\mathbb{F}$-predictable processes $\left(\theta_{t}\right)_{t \in[0, T]}$ with values in $\mathbb{R}^{d}$, integrable with respect to the semimartingale $\left(\Pi_{t}\right)_{t \in[0, T]}$ above, and such that for all $0 \leq s \leq T$, the integral process $\left(Y_{s t}(\theta)\right)_{t \in[0, T]}$ is $\mathbb{F}$-adapted and bounded from below.

Definition 2.31. The set of admissible strategies on $[0, T]$ is constituted by a convex subset $\Theta:=\Theta_{0 T} \subseteq \Xi$ satisfying the stability property: for any $A \in \mathcal{F}_{s}$ and any $\theta^{(1)}, \theta^{(2)}, \theta^{(3)} \in \Theta$ the strategy $\theta=\left(\theta_{t}\right)_{t}$ given by

$$
\theta_{t}:= \begin{cases}\theta_{t}^{(1)}, & t \in(0, s] \\ 1_{A} \theta_{t}^{(2)}+1_{A^{c}} \theta_{t}^{(3)}, & t \in(s, T]\end{cases}
$$

belongs to $\Theta$. Moreover we consider $0 \in \Theta, Y_{\text {st }}(0)=0$.

For any $s \leq t$, the set $\Theta_{\text {st }}$ of admissible strategies on $(s, t]$ is constituted by all strategies $\theta 1_{(s, t]}$ with $\theta \in \Theta$.

Clearly if $\Theta=\Xi$, then the stability property is naturally satisfied. Our choice to assume $\Theta \subseteq \Xi$ allows for a framework where it is possible to consider exogenous constrains on the applicable strategies.

Definition 2.32. For $p \in[1, \infty]$, the sets of feasible claims $\left(\mathcal{C}_{s t}^{p}\right)_{s, t}$ are defined by

$$
\mathcal{C}_{s t}^{p}:=\left\{g \in L_{p}\left(\mathcal{F}_{t}\right): \quad \exists \theta \in \Theta \text { such that } g \leq Y_{s t}(\theta)\right\} .
$$

Note that $0 \in \mathcal{C}_{s t}^{p}$.

For the risk-indifference price to be well defined, we introduce the following technical assumption, standing for this paper.

Assumption:

$$
\underset{g \in \mathcal{C}_{s t}^{p}}{\operatorname{essinf}} \rho_{s t}(g)>-\infty \quad P-\text { a.s. }
$$

Motivated by the above considerations we give the following definition.

Definition 2.33. Let $p \in[1, \infty]$. For any $s, t \in[0, T]: s \leq t$, the operator

$$
x_{s t}(X):=\underset{g \in \mathcal{C}_{s t}^{p}}{\operatorname{essinf}} \rho_{s t}(g-X)-\underset{g \in \mathcal{C}_{s t}^{p}}{\operatorname{essinf}} \rho_{s t}(g)
$$

is well-defined $P$-a.s. for all $X \in L_{p}\left(\mathcal{F}_{t}\right)$ as an $\mathcal{F}_{s}$-measurable random variable. We call $x_{s t}(X)$ the risk-indifference price of $X$ given by $\rho_{s t}$ when the operator $x_{s t}$ is considered on

$$
\operatorname{Dom}_{s t}:=\left\{X \in L_{p}\left(\mathcal{F}_{t}\right): x_{s t}(X) \in L_{p}\left(\mathcal{F}_{s}\right)\right\} .
$$

Remark 2.34. Due to the stability property of $\Theta$, the set $\left\{\rho_{s t}(g-X), g \in\right.$ $\left.\mathcal{C}_{s t}^{p}\right\}$ has the lattice property for all $X \in L_{p}\left(\mathcal{F}_{t}\right)$. 
Remark 2.35. We remark that, if the set of strategies $\Theta$ is such that $Y_{s t}(\theta) \in L_{p}\left(\mathcal{F}_{t}\right)$, for all $t$, then $Y_{s t}(\theta) \in \mathcal{C}_{s t}^{p}$. By monotonicity of the riskmeasures, we can see that, in this case, (2.20) coincides with (2.18).

Remark 2.36. We stress that if the family $\left(\rho_{s t}\right)_{s, t}$ is strong time-consistent and not normalised, then the normalisation $\check{\rho}_{s t}(X):=\rho_{s t}(X)-\rho_{s t}(0), X \in$ $L_{p}\left(\mathcal{F}_{t}\right)$, produces a family which is not strong time-consistent, but only timeconsistent. See Remark 2.12 and Remark 2.11.

It is true that the definition of the risk-indifference price operator (2.20) can be equivalently formulated by a representation in terms of both the fully-dynamic risk-measures $\left(\rho_{s t}\right)_{s, t}$ and $\left(\check{\rho}_{s t}\right)_{s, t}$. Namely, we have:

$$
\begin{aligned}
x_{s t}(X) & =\underset{g \in \mathcal{C}_{s t}^{p}}{\operatorname{essinf}} \rho_{s t}(g-X)-\underset{g \in \mathcal{C}_{s t}^{p}}{\operatorname{essinf}} \rho_{s t}(g) \\
& =\underset{g \in \mathcal{C}_{s t}^{p}}{\operatorname{essinf}} \check{\rho}_{s t}(g-X)-\underset{g \in \mathcal{C}_{s t}^{p}}{\operatorname{essinf}} \check{\rho}_{s t}(g), \quad X \in L_{p}\left(\mathcal{F}_{t}\right) .
\end{aligned}
$$

However, the first representation is in terms of a strong time-consistent family, while the second is not. As we shall see in the sequel of the paper, strong time-consistency is crucial to guarantee that the risk-indifferent prices $\left(x_{s t}\right)_{s, t}$ constitutes a convex price system. It is then not enough to work with the normalised version $\left(\check{\rho}_{s t}\right)_{s, t}$ of $\left(\rho_{s t}\right)_{s, t}$. See Remark 2.12. For this reason we focus on the first representation of the risk-indifference prices in terms of the strong time-consistent fully-dynamic risk measure $\left(\rho_{s t}\right)_{s, t}$, see Definition 2.33.

Lemma 2.37. Let $p \in[1, \infty]$.

1. If $\underset{g \in \mathcal{C}_{s t}^{p}}{\operatorname{essinf}} \rho_{s t}(g)$ belongs to $L_{p}\left(\mathcal{F}_{t}\right)$, then Dom $x_{s t}=L_{p}\left(\mathcal{F}_{t}\right)$.

2. For all $s \leq t$, we have that, for all $X \in L_{\infty}\left(\mathcal{F}_{t}\right), x_{s t}(X)$ belongs to $L_{\infty}\left(\mathcal{F}_{s}\right)$. In particular, $L_{\infty}\left(\mathcal{F}_{t}\right) \subseteq$ Dom $x_{s t}$.

Proof. For all $X \geq 0$, we have

$$
\begin{aligned}
0 \leq x_{s t}(X) & =\underset{g \in \mathcal{C}_{s t}^{p}}{\operatorname{essinf}} \rho_{s t}(g-X)-\underset{g \in \mathcal{C}_{s t}^{p}}{\operatorname{essinf}} \rho_{s t}(g) \\
& \leq \rho_{s t}(-X)-\underset{g \in \mathcal{C}_{s t}^{p}}{\operatorname{essinf}} \rho_{s t}(g) \in L_{p}\left(\mathcal{F}_{s}\right) .
\end{aligned}
$$

From the monotonicity, the convexity of $x_{s t}$, and $x_{s t}(0)=0$, we obtain that, for all $X \in L_{p}\left(\mathcal{F}_{t}\right)$,

$$
-x_{s t}(|X|) \leq-x_{s t}(-X) \leq x_{s t}(X) \leq x_{s t}(|X|) .
$$

Then $\left|x_{s t}(X)\right| \leq x_{s t}(|X|)$ and $x_{s t}(X) \in L_{p}\left(\mathcal{F}_{s}\right)$. By this we have proved 1. Now, let $X \in L_{\infty}\left(\mathcal{F}_{t}\right)$, then there are two real numbers $N, M$ such that $N \leq X \leq M$. It follows easily from the translation invariance property that for all $s, N \leq x_{s t}(X) \leq M$. This yields 2 . 
In particular we stress that, for any $p \in[1, \infty]$, the operator $x_{s t}$ in $(2.20)$ restricted to $L_{\infty}\left(\mathcal{F}_{t}\right)$ is always well-defined with values in $L_{\infty}\left(\mathcal{F}_{s}\right)$.

We give now an alternative formula for $x_{s t}$.

Lemma 2.38. For all $s \leq t$, for all $X \in L_{p}\left(\mathcal{F}_{t}\right)$, we have

$$
\underset{g \in \mathcal{C}_{s t}^{p}}{\operatorname{essinf}} \rho_{s t}(g-X)=\underset{g \in \mathcal{C}_{s t}^{\infty}}{\operatorname{essinf}} \rho_{s t}(g-X),
$$

where

$$
\mathcal{C}_{s t}^{\infty}:=\left\{g \in L_{\infty}\left(\mathcal{F}_{t}\right): \exists \theta \in \Theta \text { such that } g \leq Y_{s t}(\theta)\right\} .
$$

Then, for all $X \in L_{p}\left(\mathcal{F}_{t}\right)$,

$$
x_{s t}(X)=\underset{g \in \mathcal{C}_{s t}^{\infty}}{\operatorname{essinf}} \rho_{s t}(g-X)-\underset{g \in \mathcal{C}_{s t}^{\infty}}{\operatorname{essinf}} \rho_{s t}(g) .
$$

Proof. Since $\mathcal{C}_{s t}^{\infty} \subset \mathcal{C}_{s t}^{p}$, then we have that, for all $X \in L_{p}\left(\mathcal{F}_{t}\right)$,

$$
\underset{g \in \mathcal{C}_{s t}^{p}}{\operatorname{essinf}} \rho_{s t}(g-X) \leq \underset{g \in \mathcal{C}_{s t}^{\infty}}{\operatorname{essinf}} \rho_{s t}(g-X) .
$$

Let $g_{0} \in \mathcal{C}_{s t}^{p}$. Let $\theta \in \Theta$ such that $g_{0} \leq Y_{s t}(\theta)$. From Definition 2.30 we have that there is $C>0$ and $Y_{s t}(\theta) \geq-C$. It follows that $g^{\prime}=\sup \left(g_{0},-C\right)$ satisfies $g_{0} \leq g^{\prime} \leq Y_{s, t}(\theta)$ and $\left|g^{\prime}\right| \leq \sup \left(C,\left|g_{0}\right|\right)$. Thus $g^{\prime} \in \mathcal{C}_{s t}^{p}$ and $\rho_{s t}\left(g_{0}-\right.$ $X) \geq \rho_{s t}\left(g^{\prime}-X\right)$. The random variable $g^{\prime}$ is bounded from below and thus it is the increasing limit of the sequence $g_{n}^{\prime}=\inf \left(g^{\prime}, n\right)$. Observe that $g_{n}^{\prime} \in L_{\infty}\left(\mathcal{F}_{t}\right), g_{n}^{\prime} \leq Y_{s t}(\theta)$. The continuity from below of $\rho_{s t}$ yields $\rho_{s t}\left(g^{\prime}-X\right)=\lim _{n \rightarrow \infty} \rho_{s t}\left(g_{n}^{\prime}-X\right)$. This gives

$$
\rho_{s t}\left(g_{0}-X\right) \geq \underset{g \in \mathcal{C}_{s t}^{\infty}}{\operatorname{essinf}} \rho_{s t}(g-X) .
$$

The proof is complete.

\section{Examples.}

Risk-indifference prices are constructed from fully-dynamic risk measures $\left(\rho_{s t}\right)_{s, t}$ on $\left(L_{p}\left(\mathcal{F}_{t}\right)\right)_{t}$. As illustration, we have earlier considered $\left(\rho_{s t}\right)_{s, t}$ generated from BMO martingales and from BSDEs.

- We observe here that (2.19) is always satisfied when we consider a set of strategies $\Theta$ such that, for all $s \leq t$, there is a random variable $\xi_{t} \in L_{p}\left(\mathcal{F}_{t}\right)$ such that $Y_{s t}(\theta) \leq \xi_{t}$, for all $\theta \in \Theta$. This follows from the definition of $\mathcal{C}_{s t}^{p}$ (Definition 2.32) and the monotonicity of $\rho_{s t}$. Also observe that, for these strategies, Lemma 2.37 point 1 . holds. 
- Let us consider the fully-dynamic risk measures from the BMO martingales as constructed in Proposition 2.23. Let $p^{\prime}$ be such that $p(K)<$ $p^{\prime}<p<\infty$, where $p(K)$ is defined in Proposition 2.23. Consider $\Theta$ to be the set of strategies so that, for all $s \leq t$, there exists a random variable $\xi_{t} \in L_{p^{\prime}}\left(\mathcal{F}_{t}\right)$ and $\xi_{t} \notin L_{p}\left(\mathcal{F}_{t}\right)$, such that $Y_{s t}(\theta) \leq \xi_{t}$, for all $\theta \in \Theta$. From the arguments of Lemma 2.22 and Proposition 2.23, we see that $\rho_{s t}\left(\xi_{t}\right) \in L_{p^{\prime}}\left(\mathcal{F}_{s}\right)$. From the monotonicity, we see that $(2.19)$ is satisfied. However, in this case, Lemma 2.37 point 1 . does not hold.

\subsubsection{Properties of the risk-indifference price operator $x_{s t}$}

Hereafter we study the properties of the risk-indifference price operator $x_{s t}$ for fixed $s \leq t$.

Proposition 2.39. The operator $x_{s t}(X), X \in L_{p}\left(\mathcal{F}_{t}\right)$, is monotone, convex, it has the projection property, it is weak $\mathcal{F}_{s}$-homogeneous, and it is continuous from above.

Proof. The monotonicity of $x_{s t}$ follows from the corresponding property of $\rho_{s t}$. The projection property of $x_{s t}$ follows from the $\mathcal{F}_{s}$-translation invariance of $\rho_{s t}$ and $x_{s t}(0)=0$. For the convexity of $x_{s t}$ we argue as follows. First of all note that $\mathcal{C}_{s t}^{p}$ is convex, see the convexity of $\Theta$ (Definition 2.31) and Definition 2.32. Therefore, for all $g_{1}$ and $g_{2}$ in $\mathcal{C}_{s t}^{p}, \lambda g_{1}+(1-\lambda) g_{2} \in \mathcal{C}_{s t}^{p}$. Thus

$$
\inf _{g \in \mathcal{C}_{s t}^{p}} \rho_{s t}\left(g-[\lambda X+(1-\lambda Y]) \leq \inf _{g_{1}, g_{2} \in \mathcal{C}_{s t}^{p}} \rho_{s t}\left[\lambda\left(g_{1}-X\right)+(1-\lambda)\left(g_{2}-Y\right)\right] .\right.
$$

The convexity of $\rho_{s t}$ yields that

$$
\inf _{g \in \mathcal{C}_{s t}^{p}} \rho_{s t}\left(g-[\lambda X+(1-\lambda Y]) \leq \inf _{g_{1}, g_{2} \in \mathcal{C}_{s t}^{p}}\left[\lambda \rho_{s t}\left(g_{1}-X\right)+(1-\lambda) \rho_{s t}\left(g_{2}-Y\right)\right] .\right.
$$

This gives the convexity of $x_{s t}$. The weak $\mathcal{F}_{s}$-homogeneity is justified as follows. Let $A \in \mathcal{F}_{s}$, then

$$
\begin{aligned}
& 1_{A} x_{s t}(X)=1_{A}\left[\underset{g \in \mathcal{C}_{s t}^{p}}{\operatorname{essinf}} \rho_{s t}(g-X)-\underset{g \in \mathcal{C}_{s t}^{p}}{\operatorname{essinf}} \rho_{s t}(g)\right] \\
& =\underset{g \in \mathcal{C}_{s t}^{p}}{\operatorname{essinf}} 1_{A} \rho_{s t}(g-X)-\underset{g \in \mathcal{C}_{s t}^{p}}{\operatorname{essinf}} 1_{A} \rho_{s t}(g) \\
& =\underset{g \in \mathcal{C}_{s t}^{p}}{\operatorname{essinf}} 1_{A} \rho_{s t}\left(g-1_{A} X\right)-\underset{g \in \mathcal{C}_{s t}^{p}}{\operatorname{essinf}} 1_{A} \rho_{s t}(g) \\
& =1_{A} x_{s t}\left(1_{A} X\right),
\end{aligned}
$$

where the weak $\mathcal{F}_{s}$-homogeneity of $\rho_{s t}$ justifies the third inequality. In fact, $1_{A} \rho_{s t}(g-X)=1_{A} \rho_{s t}\left(1_{A}(g-X)\right)=1_{A} \rho_{s t}\left(1_{A}\left(g-1_{A} X\right)\right)=1_{A} \rho_{s t}\left(g-1_{A} X\right)$.

Finally, recall that for every $0 \leq s \leq t \leq T$, the risk measure $\rho_{s t}$ is continuous from below. Let $\left(X_{n}\right)_{n}$ in $L_{p}\left(\mathcal{F}_{t}\right)$ be a sequence decreasing to $X \in L_{p}\left(\mathcal{F}_{t}\right)$. 
Then we have that $\rho_{s t}(g-X)$ is the decreasing limit of $\rho_{s t}\left(g-X_{n}\right)$, for $n \rightarrow \infty$. Hence,

$$
\begin{aligned}
x_{s t}(X) & =\operatorname{essinf}_{g \in \mathcal{C}_{s t}^{p}} \inf _{n} \rho_{s t}\left(g-X_{n}\right)-\underset{g \in \mathcal{C}_{s t}^{p}}{\operatorname{essinf}} \rho_{s t}(g) \\
& =\inf _{n}\left[\underset{g \in \mathcal{C}_{s t}^{p}}{\operatorname{essinf}} \rho_{s t}\left(g-X_{n}\right)-\underset{g \in \mathcal{C}_{s t}^{p}}{\operatorname{essinf}} \rho_{s t}(g)\right] \\
& =\inf _{n} x_{s t}\left(X_{n}\right) .
\end{aligned}
$$

The monotonicity of $x_{s t}$ implies that $x_{s t}(X)$ is the decreasing limit of $x_{s t}\left(X_{n}\right)$. The continuity from above of $x_{s t}$ is then proved.

In the last part of this subsection we study the Fatou property for the riskindifference price operator $x_{s t}$. For this we shall distinguish the two cases when $p=\infty$ and $p \in[1, \infty)$. We have to recall that

$$
L_{\infty}\left(\mathcal{F}_{t}\right) \subseteq D_{o m x_{s t}} \subseteq L_{p}\left(\mathcal{F}_{t}\right), \quad p \geq 1,
$$

from Lemma 2.37 item 2.

Proposition 2.40. Let $p=\infty$. The risk-indifference price operator $x_{s t}$ admits the following representation

$$
x_{s t}(X)=\operatorname{esssup}_{Q \ll P, Q_{\mid \mathcal{F}_{s}}=P}\left(E_{Q}\left(X \mid \mathcal{F}_{s}\right)-\gamma_{s t}(Q)\right), \quad X \in L_{\infty}\left(\mathcal{F}_{t}\right),
$$

where $\gamma_{s t}(Q)$ is the minimal penalty:

$$
\gamma_{s t}(Q)=\operatorname{esssup}_{X \in L_{\infty}\left(\mathcal{F}_{t}\right)}\left(E_{Q}\left(X \mid \mathcal{F}_{s}\right)-x_{s t}(X)\right) .
$$

In particular $x_{s t}$ has the Fatou property and is continuous on $L_{\infty}\left(\mathcal{F}_{t}\right)$.

Proof. The representation follows from the continuity from above, see Proposition 2.39. Indeed we refer to the dual representation for conditional risk measures as in [22] or in [8]. This dual representation is written for the conditional risk measure $x_{s t}(-X)$. The second assertion follows from the representation (2.23) itself and [22].

Remark 2.41. If the operator $x_{s t}$ was considered on a Fréchet lattice $L_{t} \supseteq$ $L_{\infty}\left(\mathcal{F}_{t}\right)$, then one could obtain the Fatou property by applying the extension of the Namioka-Klee theorem, see [5], to the functional $E\left[x_{s t}(X)\right], X \in L_{t}$. We have to remark that Dom $x_{\text {st }}$ is not a Fréchet lattice, in fact it is not complete and also in general we have $\Theta \subseteq \Xi$ hence it may not even be a vector space. 
As Remark 2.41 shows the study of the Fatou property (and also timeconsistency as we shall see later) is delicate and the major issues are related to the domain of the operators involved. This is again noticed in e.g. [27] in the context of forward utilities.

In view of the comments above, we now propose a different approach to study the Fatou property for $p \in[1, \infty)$. For all $s, t \in[0, T]: s \leq t$, we shall introduce an extension of the operator $x_{s t}$ by constructing an adequate extension of $\rho_{s t}$. For this, when $p \in[1, \infty)$, we assume that $\rho_{0 T}$ is dominated and sensitive.

We introduce the seminorm

$$
c(X):=\sup _{Q \in \mathcal{Q}} E_{Q}(|X|),
$$

for all $\mathcal{F}_{T}$ measurable random variables $X$ and $\mathcal{Q}:=\left\{Q \sim P: \alpha_{0 T}(Q)<\right.$ $\infty$, where $\alpha_{0 T}$ is the minimal penalty of $\rho_{0 T}$ (see Proposition 2.19). We observe that

$$
c(X)=0 \quad \Longleftrightarrow \quad X=0 \quad P-a . s .
$$

This follows directly from $Q \sim P$ in the definition of $\mathcal{Q}$. Then $c$ induces a relationship of equivalence among random variables.

Definition 2.42. For all $t$, we define $\mathcal{L}_{t}^{c}$ to be the completion, with respect to the seminorm $c$, of the set of essentially bounded $\mathcal{F}_{t}$-measurable random variables. We define $L_{t}^{c}:=\mathcal{L}_{t}^{c} / \sim$.

The space $L_{t}^{c}$ is a Banach space with norm $c$.

Lemma 2.43. For all $t$, the following relationship holds for all $Q \in \mathcal{Q}$ :

$$
L_{p}\left(\mathcal{F}_{t}, P\right) \subseteq L_{t}^{c} \subseteq L_{1}\left(\mathcal{F}_{t}, Q\right)
$$

Proof. The relationship is directly proved from Proposition 2.19, in fact $E_{Q}(|X|) \leq c(X) \leq K\|X\|_{p}$, for all $Q \in \mathcal{Q}$.

In the sequel, we extend the risk measure $\rho_{s t}$ to obtain the map:

$$
\tilde{\rho}_{s t}: L_{t}^{c} \longrightarrow L_{s}^{c}
$$

and then we extend the corresponding risk-indifference price $x_{s t}$ as a map:

$$
x_{s t}: L_{t}^{c} \longrightarrow L_{s}^{c} .
$$

This operator allows us to study the Fatou property. Also we shall see that the extensions above are instrumental in the study of time-consistency for the price system.

In the literature we find other extensions of risk-measures to a larger domain of the type $L_{t}^{c}$. See e.g. [35], [40]. We observe that these are related to 
risk-measures with values in $\mathbb{R}$ or $(-\infty, \infty]$. In this paper, we deal with the extension of the whole family of risk measures $\left(\rho_{s t}\right)_{s, t}$ as operators: $\rho_{s t}: L_{p}\left(\mathcal{F}_{t}\right) \longrightarrow L_{p}\left(\mathcal{F}_{s}\right)$. We perform such extensions substantially under the assumptions on the risk measure $\rho_{0 T}$ to be dominated and sensitive and the use of the strong time-consistency of the original family $\left(\rho_{s t}\right)_{s, t}$. We highlight the crucial role of the strong time-consistency in this process. As a result, we obtain that the extended family $\left(\tilde{\rho}_{s t}\right)_{s, t}$ is also strong timeconsistent. In turn this is important to obtain the time-consistency of the risk-indifference prices. We shall detail these arguments in the sequel.

\subsubsection{Construction of the extended fully-dynamic risk-measure}

The construction of the extension of $\rho_{s t}$ is engaging and it requires several steps. First of all we have the following lemmas.

Lemma 2.44. For fixed $t \in[0, T]$, consider a convex risk measure $\psi_{t T}$ : $L_{\infty}\left(\mathcal{F}_{T}\right) \longrightarrow L_{\infty}\left(\mathcal{F}_{t}\right)$ continuous from below. Let $\beta_{t T}$ be its minimal penalty. Assume that there exists a probability measure $Q \sim P$ such that $E_{Q}\left(\beta_{t T}(Q)\right)<$ $\infty$. Then the following representation holds

$$
\psi_{t T}(X)=\operatorname{esssup}_{R \sim P, \beta_{t T}(R) \in L_{\infty}\left(\mathcal{F}_{t}\right)}\left(E_{R}\left(-X \mid \mathcal{F}_{t}\right)-\beta_{t T}(R)\right) .
$$

Proof. The proof is organised in steps.

Step 1. Consider $\Phi_{t}(X):=-\psi_{t T}(X), X \in L_{\infty}\left(\mathcal{F}_{T}\right)$, and $\alpha_{t}(R):=-\beta_{t T}(R)$, $\overline{R \sim P}$. Then the mapping $\Phi_{t}$ satisfies the property $\left.\mathbf{I}\right)$ in Theorem 3.1 of [33]. This result presents a number of equivalent statements and then property II) is true. From the proof that II) implies I) (see Appendix in [33]) we have that for all $\varepsilon>0$ there is a probability measure $\tilde{Q} \sim P$ such that $\alpha_{t}(\tilde{Q})+\varepsilon \geq-\Phi_{t}(0)$. On the other hand, we have that

$$
-\Phi_{t}(0)=\psi_{t T}(0) \geq-\beta_{t T}(\tilde{Q})=\alpha_{t}(\tilde{Q}) .
$$

This proves that there exists $\tilde{Q} \sim P$ such that

$$
\beta_{t T}(\tilde{Q})=-\alpha_{t}(\tilde{Q}) \text { belongs to } L_{\infty}\left(\mathcal{F}_{t}\right) .
$$

The property of the minimal penalty allow us to consider $\tilde{Q}_{\mid \mathcal{F}_{t}}=P$.

Step 2. Let $X \in L_{\infty}\left(\mathcal{F}_{t}\right)$. For all $R \sim P$ such that $R_{\mid \mathcal{F}_{t}}=P$ let

$$
A=\left\{E_{R}\left(-X \mid \mathcal{F}_{t}\right)-\beta_{t T}(R)>E_{\tilde{Q}}\left(-X \mid \mathcal{F}_{t}\right)-\beta_{t T}(\tilde{Q})\right\} .
$$

Let $\tilde{R}$ be defined by

$$
\frac{d \tilde{R}}{d P}=1_{A} \frac{d R}{d P}+1_{A^{c}} \frac{d \tilde{Q}}{d P} .
$$

Then we have that $\tilde{R} \sim P, \tilde{R}_{\mid \mathcal{F}_{t}}=P$, and $\beta_{t T}(\tilde{R})=1_{A} \beta_{t T}(R)+1_{A^{c}} \beta_{t T}(\tilde{Q})$. From the definition of the event $A$, we can see that $\beta_{t T}(R) 1_{A} \leq \beta_{t T}(\tilde{Q}) 1_{A}+$ 
$2\|X\|_{\infty} 1_{A}$. On the other hand we have $\beta_{t T}(R) \geq-\psi_{t T}(0)$. Thus we conclude that $\beta_{t T}(\tilde{R}) \in L_{\infty}\left(\mathcal{F}_{t}\right)$ and $E_{\tilde{R}}\left(X \mid \mathcal{F}_{t}\right)-\beta_{t T}(\tilde{R}) \geq E_{R}\left(X \mid \mathcal{F}_{t}\right)-\beta_{t T}(R)$. By this we have obtained representation (2.24).

Lemma 2.45. Set $p \in[1, \infty)$. Let $\left(\rho_{s t}\right)_{s, t}$ be a strong time-consistent fullydynamic risk measure such that $\rho_{0 T}$ is dominated and sensitive. For all $Q \in \mathcal{Q}$, for all $s \in[0, T]$, we have that the minimal penalties $\left(\alpha_{s t}\right)_{s, t}$ satisfy the following:

$$
\alpha_{0 s}(Q) \in \mathbb{R} \quad \text { and } \quad E_{Q}\left(\alpha_{s T}(Q)\right) \in \mathbb{R} .
$$

Proof. The minimal penalties $\left(\alpha_{s t}\right)_{s, t}$ satisfy the cocycle condition:

$$
\alpha_{0 T}(Q)=\alpha_{0 s}(Q)+E_{Q}\left(\alpha_{s T}(Q)\right), \quad Q \in \mathcal{Q},
$$

see [10] (Remark 2.12). Furthermore, it follows from the definition of minimal penalty that

$$
\alpha_{0 s}(Q) \geq-\rho_{0 s}(0) \quad \text { and } \quad \alpha_{s T}(Q) \geq-\rho_{s T}(0) .
$$

By hypothesis we have that $\rho_{s T}(0) \in L_{p}\left(\mathcal{F}_{s}\right)$ and, from Proposition 2.19, we have that $\frac{d Q}{d P} \in L_{q}\left(\mathcal{F}_{T}\right)$, with $q=p(p-1)^{-1}$. Thus $E_{Q}\left(-\rho_{s T}(0)\right) \in \mathbb{R}$. The result follows from $\alpha_{0 T}(Q)<\infty$ and the cocycle condition (2.25).

We introduce the following sets of probability measures for all $s \leq t$ on $\left(\Omega, \mathcal{F}_{t}\right)$ :

$$
\mathcal{P}_{s t}:=\left\{R \sim P: R_{\mid \mathcal{F}_{s}}=P \text { and } \alpha_{s t}(R) \in L_{p}\left(\mathcal{F}_{s}\right)\right\}
$$

and

$$
\widetilde{\mathcal{P}}_{s t}:=\left\{R \sim P: R_{\mid \mathcal{F}_{s}}=P \text { and } \sup _{Q \in \mathcal{Q}} E_{Q}\left(\alpha_{s t}(R)\right)<\infty\right\}
$$

We also remark immediately that

$$
\mathcal{P}_{s t} \subseteq \widetilde{\mathcal{P}}_{s t}=\left\{R \sim P: R_{\mid \mathcal{F}_{s}}=P \text { and } \sup _{Q \in \mathcal{Q}} E_{Q}\left(\left|\alpha_{s t}(R)\right|\right)<\infty\right\} .
$$

In fact, for $R \in \widetilde{\mathcal{P}}_{s t}$,we have that $\alpha_{s t}(R) \geq-\rho_{s t}(0)$. Then $\left|\alpha_{s t}(R)\right| \leq$ $\alpha_{s t}(R)+2\left|\rho_{s t}(0)\right|$. We remind that $\rho_{s t}(0) \in L_{p}\left(\mathcal{F}_{t}\right)$ and $\left\|\frac{d Q}{d P}\right\|_{L_{q}} \leq K$ for all $Q \in \mathcal{Q}$, see Proposition 2.19. Then we conclude that both relations hold.

Proposition 2.46. Set $p \in[1, \infty)$. Let $\left(\rho_{s t}\right)_{s, t}$ be a strong time-consistent fully-dynamic risk measure such that $\rho_{0 T}$ is dominated and sensitive. Then we have that

1. for all $t \in[0, T]$, the risk measure $\rho_{0 t}$ is dominated and sensitive,

2. for all $t \in[0, T]: s \leq t$, the following representation holds:

$$
\rho_{s t}(X)=\underset{R \in \mathcal{P}_{s t}}{\operatorname{esssup}}\left(E_{R}\left(-X \mid \mathcal{F}_{s}\right)-\alpha_{s t}(R)\right), \quad X \in L_{p}\left(\mathcal{F}_{t}\right),
$$


3. for all $Q \in \mathcal{Q}$ and $R \in \widetilde{\mathcal{P}}_{\text {st }}$, there exists $h \in L_{q}\left(\mathcal{F}_{t}\right):\|h\|_{q} \leq K$, with $q=p(p-1)^{-1}$, such that

$$
E_{Q}\left(E_{R}\left(X \mid \mathcal{F}_{s}\right)\right)=E(h X), \quad X \in L_{p}\left(\mathcal{F}_{t}\right),
$$

4. for all $t \in[0, T]: s \leq t$, the following representation holds:

$$
\rho_{s t}(X)=\underset{R \in \widetilde{\mathcal{P}}_{s t}}{\operatorname{esssup}}\left(E_{R}\left(-X \mid \mathcal{F}_{s}\right)-\alpha_{s t}(R)\right), \quad X \in L_{p}\left(\mathcal{F}_{t}\right) .
$$

Proof.

1. For all $Z \in L_{p}\left(\mathcal{F}_{t}\right)$ we have $\rho_{0 T}(Z)=\rho_{0 t}\left(-\rho_{t T}(Z)\right)=\rho_{0 t}\left(Z-\rho_{t T}(0)\right)$. By hypothesis, $\rho_{t T}(0) \in L_{p}\left(\mathcal{F}_{t}\right)$, thus for all $Y \in L_{p}\left(\mathcal{F}_{t}\right)$ we have $\rho_{0 t}(Y)=\rho_{0 T}\left(Y+\rho_{t T}(0)\right)$. This shows that $\rho_{0 t}$ is dominated. From Lemma 2.45, $\rho_{0 t}$ is sensitive. This completes the proof of item 1.

2. and 3. The proofs of items 2 and 3 proceed together, first proving the result in item 2 for $X \in L_{\infty}\left(\mathcal{F}_{t}\right)$, then item 3 , and finally, item 2 for $X \in L_{p}\left(\mathcal{F}_{t}\right)$. The argument is split in steps.

Step 1. Let $0 \leq s \leq t \leq T$. Define

$$
\breve{\rho}_{s t}(X):=\rho_{s t}(X)-\rho_{s t}(0), \quad X \in L_{p}\left(\mathcal{F}_{t}\right) .
$$

The translation invariance and the monotonicity of $\rho_{s t}$ imply that

$$
\breve{\rho}_{s t}: L_{\infty}\left(\mathcal{F}_{t}\right) \longrightarrow L_{\infty}\left(\mathcal{F}_{s}\right) .
$$

The minimal penalty associated to the restriction of $\breve{\rho}_{s t}$ to $L_{\infty}\left(\mathcal{F}_{t}\right)$ is

$$
\breve{\alpha}_{s t}(Q)=\operatorname{esssup}_{X \in L_{\infty}\left(\mathcal{F}_{t}\right)}\left(E_{Q}\left(-X \mid \mathcal{F}_{s}\right)-\breve{\rho}_{s t}(X)\right) .
$$

for all $Q \sim P$. Thus

$$
\breve{\alpha}_{s t}(Q)=\operatorname{esssup}_{X \in L_{\infty}\left(\mathcal{F}_{t}\right)}\left(E_{Q}\left(-X \mid \mathcal{F}_{s}\right)-\rho_{s t}(X)\right)+\rho_{s t}(0) .
$$

Then Lemma 2.9 yields

$$
\breve{\alpha}_{s t}(Q)=\alpha_{s t}(Q)+\rho_{s t}(0) .
$$

From (2.30) and Lemma 2.45 we obtain that $E_{Q}\left(\breve{\alpha}_{s t}(Q)\right)<\infty$ for all $Q$ such that $E\left(\alpha_{s t}(Q)\right)<\infty$. From Lemma 2.44 we have that the risk measure $\breve{\rho}_{s t}$ satisfies the representation (2.24). This, together with (2.29) and $(2.30)$ and $\rho_{s t}(0) \in L_{p}\left(\mathcal{F}_{s}\right)$, proves that $(2.27)$ is satisfied for all $X \in L_{\infty}\left(\mathcal{F}_{t}\right)$. 
Step 2. Let $Q \in \mathcal{Q}$ and $R \in \tilde{\mathcal{P}}_{\text {st }}$. For all $X \in L_{p}\left(\mathcal{F}_{t}\right)$ we have

$$
E_{Q}\left(E_{R}\left(-X \mid \mathcal{F}_{s}\right)\right)-\alpha_{0 s}(Q)-E_{Q}\left(\alpha_{s t}(R)\right) \leq \rho_{0 s}\left(-\rho_{s t}(X)\right)=\rho_{0 t}(X)
$$

From Step 1, $\rho_{0 t}$ is dominated: $\rho_{0 t}(X) \leq K\|X\|_{p}+C$. Moreover, $\alpha_{0 s}(Q)<\infty$ and $\sup _{Q \in \mathcal{Q}} E_{Q}\left(\alpha_{s t}(R)\right)<\infty$. Thus there is some $\kappa \in \mathbb{R}$ such that

$$
E_{Q}\left(E_{R}\left(-X \mid \mathcal{F}_{s}\right)\right) \leq K\|X\|_{p}+\kappa, \quad X \in L_{p}\left(\mathcal{F}_{t}\right) .
$$

This proves item 3 .

Step 3. Let $Q \in \mathcal{Q}$. The functional $\phi(X):=E_{Q}\left(\rho_{s t}(X)\right), X \in L_{p}\left(\mathcal{F}_{t}\right)$,

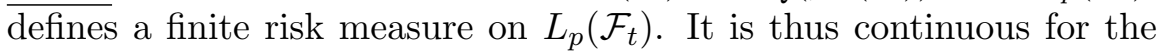
$L_{p}$-norm, see [26]. From the definition of the minimal penalty we have that

$$
\rho_{s t}(X) \geq \underset{R \in \mathcal{P}_{s t}}{\operatorname{esssup}}\left(E_{R}\left(-X \mid \mathcal{F}_{s}\right)-\alpha_{s t}(R)\right) .
$$

Furthermore, for any given $X$, the set

$$
\left\{E_{R}\left(-X \mid \mathcal{F}_{s}\right)-\alpha_{s t}(R), R \in \mathcal{P}_{s t}\right\}
$$

is a lattice upward directed. Thus, in view of Proposition VI1.1 in [38], in order to prove $(2.27)$ for all $X \in L_{p}\left(\mathcal{F}_{t}\right)$, it is enough to prove that

$$
\begin{aligned}
\phi(X): & =E_{Q}\left(\rho_{s t}(X)\right) \\
& =\sup _{R \in \mathcal{P}_{s t}}\left(E _ { Q } \left(E_{R}\left(-X \mid \mathcal{F}_{s}\right)-E_{Q}\left(\alpha_{s t}(R)\right), \quad X \in L_{p}\left(\mathcal{F}_{t}\right) .\right.\right.
\end{aligned}
$$

From Step 1, we already know that (2.31) is satisfied for all $X \in$ $L_{\infty}\left(\mathcal{F}_{t}\right)$. Observe that $\phi$ is continuous in the $L_{p}$-norm. Moreover, the continuity of the right-hand side of $(2.31)$ in the $L_{p}$-norm follows from Step 2 and the inclusion $\mathcal{P}_{s t} \subseteq \tilde{\mathcal{P}}_{s t}$. This ends the proof of item 2 .

4. The representation (2.28) follows directly from (2.27) and the observation that $\mathcal{P}_{s t} \subseteq \tilde{\mathcal{P}}_{s t}$.

By this the proof is complete.

\section{Lemma 2.47.}

1. For all $0 \leq r \leq s \leq t \leq T$, for all $R \in \tilde{\mathcal{P}}_{\text {st }}$, there is $S \in \tilde{\mathcal{P}}_{s T}$, such that $R$ is the restriction of $S$ to $\mathcal{F}_{t}$.

2. For all $0 \leq r \leq s \leq t \leq T, \tilde{\mathcal{P}}_{r t} \subset \tilde{\mathcal{P}}_{r s}$, which means that the restriction to $\mathcal{F}_{s}$ of an element of $\tilde{\mathcal{P}}_{r t}$, belongs to $\tilde{\mathcal{P}}_{r s}$. 
Proof.

1. $R \in \tilde{\mathcal{P}}_{s t}$, let $\tilde{Q} \in \mathcal{P}_{t T}$. Let $S$ be the probability measure equivalent with $P$ such that

$$
\frac{d S}{d P}=\frac{d R}{d P} \frac{\frac{d \tilde{Q}}{d P}}{E\left(\frac{d \tilde{Q}}{d P} \mid \mathcal{F}_{t}\right)} .
$$

In particular the restriction of $S$ to $\mathcal{F}_{t}$ is equal to $R$. It follows from the definition of $S$ and the properties of the minimal penalty that

$$
\alpha_{s T}(S)=\alpha_{s t}(R)+E_{R}\left(\alpha_{t T}(\tilde{Q}) \mid \mathcal{F}_{s}\right) .
$$

Thus

$$
\sup _{Q \in \mathcal{Q}} E_{Q}\left(\alpha_{s T}(S)\right) \leq \sup _{Q \in \mathcal{Q}} E_{Q}\left(\alpha_{s t}(R)\right)+\sup _{Q \in \mathcal{Q}} E_{Q}\left[E_{R}\left(\alpha_{t T}\left(\tilde{Q} \mid \mathcal{F}_{s}\right)\right] .\right.
$$

From item 3 of Proposition 2.46, we have that, for every $Q \in \mathcal{Q}$, there is $h \in L_{q}\left(\mathcal{F}_{t}\right)$ with $\|h\|_{q} \leq K$ such that, for all $X$ in $L_{p}\left(\mathcal{F}_{t}\right)$, $E_{Q}\left[E_{R}\left(X \mid \mathcal{F}_{s}\right)\right]=E(h X) \leq K\|X\|_{\tilde{p}}$. By definition of $\tilde{\mathcal{P}}_{s t}$ and $\mathcal{P}_{t, T}$ and using (2.32), we have that $S \in \tilde{\mathcal{P}}_{s T}$.

2. Let $R \in \tilde{\mathcal{P}}_{r t}$. It follows from the definition of the minimal penalty that $\alpha_{s t}(R) \geq-\rho_{s t}(0)$. We deduce then from the cocycle condition that

$$
\alpha_{r s}(R) \leq \alpha_{r t}(R)+E_{R}\left(\rho_{s t}(0) \mid \mathcal{F}_{r}\right)
$$

where $\rho_{s t}(0)$ belongs to $L_{p}\left(\mathcal{F}_{s}\right)$. Then, from Proposition 2.46 item 3, we obtain $\sup _{Q \in \mathcal{Q}} E_{Q}\left(\alpha_{r s}(R)\right)<\infty$. This gives the result.

The proof is complete.

We will now extend $\rho_{s t}$ to $L_{t}^{c}$ for every $0 \leq s \leq t \leq T$. We first prove the following result.

Proposition 2.48. Let $0=s_{0}<s_{1}<\ldots<s_{n}=T$. For all $Q_{i} \in \tilde{\mathcal{P}}_{s_{i} s_{i+1}}$ with $i=0, \ldots, n-1$, let $Q$ be the unique probability measure on $\mathcal{F}_{T}$ such that

$$
E_{Q}(X)=E_{Q_{0}}\left(E_{Q_{1}}\left(\cdots E_{Q_{n-1}}\left(X \mid \mathcal{F}_{s_{n-1}}\right) \cdots \mid \mathcal{F}_{s_{1}}\right)\right), \quad X \in L_{\infty}\left(\mathcal{F}_{T}\right) .
$$

Then $Q$ belongs to $\mathcal{Q}$.

Proof. For $n=1$ observe that $\tilde{\mathcal{P}}_{0 T}=\mathcal{Q}$. We prove the result by induction for $n \geq 2$.

Step 1. For $n=2$, we have $0=s_{0}<s_{1}<s_{2}=T$ and $E_{Q}(X)=$ $\bar{E}_{Q_{0}}\left(E_{Q_{1}}\left(X \mid \mathcal{F}_{s_{1}}\right)\right)$, for any $X \in L_{\infty}\left(\mathcal{F}_{T}\right)$. From the cocycle condition and the properties of the minimal penalty, it is

$$
\alpha_{0 T}(Q)=\alpha_{0 s_{1}}\left(Q_{0}\right)+E_{Q_{0}}\left(\alpha_{s_{1} T}\left(Q_{1}\right)\right) .
$$


By assumption the probability measure $Q_{0} \in \tilde{\mathcal{P}}_{0 s_{1}}$. From item 1 of Lemma 2.47 we can see that $Q_{0}$ is the restriction to $\mathcal{F}_{s_{1}}$ of an element of $\mathcal{Q}$. Then we easily see also that $\alpha_{0 T}(Q)<\infty$ from the definition of $\tilde{\mathcal{P}}_{s_{1} T}$. Thus $Q \in \mathcal{Q}$. Step 2. We assume that the result holds for $n$ and we prove it for $n+1$. $\overline{\text { Let } 0}=s_{0}<s_{1}<\ldots<s_{n}<s_{n+1}=T$. From the induction hypothesis $Q_{n-1} \in \tilde{\mathcal{P}}_{s_{n-1} s_{n}}$. From item 1 of Lemma 2.47 we obtain that $Q_{n-1}$ is the restriction to $\mathcal{F}_{s_{n}}$ of an element $R_{n-1} \in \tilde{\mathcal{P}}_{s_{n-1} T}$. Then by induction we can see that the probability measure $R$ on $\mathcal{F}_{T}$ defined by

$$
E_{R}(X)=E_{Q_{0}}\left(E_{Q_{1}}\left(\cdots E_{R_{n-1}}\left(X \mid \mathcal{F}_{s_{n-1}}\right) \cdots \mid \mathcal{F}_{s_{1}}\right)\right)
$$

for all $X$ in $L_{\infty}\left(\mathcal{F}_{T}\right)$, belongs to $\mathcal{Q}$. From item 2 of Lemma 2.47, the restriction $\tilde{R}$ of $R$ to $\mathcal{F}_{s_{n}}$ belongs to $\tilde{\mathcal{P}}_{0 s_{n}}$ and

$$
E_{Q}(X)=E_{Q_{0}}\left(E_{Q_{1}}\left(\cdots E_{Q_{n}}\left(X \mid \mathcal{F}_{s_{n}}\right) \cdots \mid \mathcal{F}_{s_{1}}\right)=E_{\tilde{R}}\left(E_{Q_{n}}\left(X \mid \mathcal{F}_{s_{n}}\right) .\right.\right.
$$

The result follows then from Step 1.

Theorem 2.49. Set $p \in[1, \infty)$. Let $\left(\rho_{s t}\right)_{s, t}$ be a strong time-consistent fully-dynamic risk measure on $\left(L_{p}\left(\mathcal{F}_{t}\right)\right)_{t}$ such that $\rho_{0 T}$ is dominated and sensitive.

1. For all $s \leq t$, the risk measure $\rho_{s t}$ admits a unique extension $\tilde{\rho}_{s t}$ : $L_{t}^{c} \rightarrow L_{s}^{c}$ such that

$$
c\left(\left|\tilde{\rho}_{s t}(X)-\tilde{\rho}_{s t}(Y)\right|\right) \leq c(|X-Y|), \quad X, Y \in L_{t}^{c} .
$$

2. The extension $\tilde{\rho}_{\text {st }}$ admits the following representation

$$
\tilde{\rho}_{s t}(X)=\underset{R \in \tilde{\mathcal{P}}_{s t}}{\operatorname{esssup}}\left(E_{R}\left(-X \mid \mathcal{F}_{s}\right)-\alpha_{s, t}(R)\right), \quad X \in L_{t}^{c}
$$

Proof.

1. Let $X, Y \in L_{\infty}\left(\mathcal{F}_{t}\right)$. Consider $A \in \mathcal{F}_{s}$ such that $\left|\rho_{s t}(X)-\rho_{s t}(Y)\right|=$ $\left[\rho_{s t}(X)-\rho_{s t}(Y)\right] 1_{A}+\left[\rho_{s t}(Y)-\rho_{s t}(X)\right] 1_{A^{c}}$. From (2.28) we get

$$
\left|\rho_{s t}(X)-\rho_{s t}(Y)\right| \leq \underset{R \in \tilde{\mathcal{P}}_{s t}}{\operatorname{esssup}} E_{R}\left(|X-Y| \mid \mathcal{F}_{s}\right) .
$$

For all $X, Y \in L_{\infty}\left(\mathcal{F}_{t}\right)$, the set $\left\{E_{R}\left(|X-Y| \mid \mathcal{F}_{s}\right), R \in \tilde{\mathcal{P}}_{s t}\right\}$ satisfies the lattice property. It follows that, for all $Q \in \mathcal{Q}$,

$$
E_{Q}\left(\underset{R \in \tilde{\mathcal{P}}_{s t}}{\operatorname{esssup}} E_{R}\left(|X-Y| \mid \mathcal{F}_{s}\right)\right)=\sup _{R \in \tilde{\mathcal{P}}_{s t}} E_{Q}\left(E_{R}\left(|X-Y| \mid \mathcal{F}_{s}\right)\right) .
$$


From equation (2.35), from item 1 of Lemma 2.47, and from Proposition 2.48 , we obtain that

$$
c\left(\left|\rho_{s t}(X)-\rho_{s t}(Y)\right|\right) \leq c(|X-Y|), \quad X, Y \in L_{\infty}\left(\mathcal{F}_{t}\right) .
$$

On the other hand, $\rho_{s t}(X) \in L_{p}\left(\mathcal{F}_{s}\right) \subset L_{s}^{c}$, for all $X \in L_{\infty}\left(\mathcal{F}_{t}\right)$. Then item 1 in the statement of the theorem follows from the density of $L_{\infty}\left(\mathcal{F}_{t}\right)$ in $L_{t}^{c}$ and from equation $(2.36)$.

2. The representation (2.34) is satisfied for all $X \in L_{\infty}\left(\mathcal{F}_{t}\right)$, see item 4 of Proposition 2.46. Item 1 of the theorem provides the continuity of $\tilde{\rho}_{s t}$ for the c norm. Moreover, from Lemma 2.47 item 1 and Proposition 2.48 , we have continuity for the c norm of the right-hand side of (2.34). By continuity, we can then see that (2.34) is satisfied for all $X$ in $L_{t}^{c}$.

This completes the proof.

Corollary 2.50. The family $\left(\tilde{\rho}_{s t}\right)_{s, t}$ is a strong time-consistent dynamic risk measure on $\left(L_{t}^{c}\right)$. Furthermore $\tilde{\rho}_{0 T}$ is dominated by $\sup _{Q \in \mathcal{Q}} E_{Q}(-X)$.

Proof. The properties of monotonicity, translation invariance, and strong time-consistency for $\left(\tilde{\rho}_{s t}\right)_{s, t}$ follow from (2.33) and the corresponding properties for $\left(\rho_{s t}\right)_{s, t}$ on $\left(L_{p}\left(\mathcal{F}_{t}\right)\right)_{t}$. The domination by $\sup _{Q \in \mathcal{Q}} E_{Q}(-X)$ follows from the representation (2.34) of $\tilde{\rho}_{0 T}$ and the observation that $\tilde{\mathcal{P}}_{0 T}=\mathcal{Q}$.

\subsubsection{The extended risk-indifference prices}

Now that we have the extension $\left(\tilde{\rho}_{s t}\right)_{s, t}$ on $\left(L_{t}^{c}\right)_{t}$, we can extend the price system $\left(x_{s t}\right)_{s, t}$ on $\left(L_{t}^{c}\right)_{t}$. Note that we shall keep the same notation $x_{s t}$ also for the corresponding extended price operator.

Definition 2.51. Set $p \in[1, \infty)$. Let $\left(\rho_{s t}\right)_{s, t}$ be a strong time-consistent, fully-dynamic risk measure on $\left(L_{p}\left(\mathcal{F}_{t}\right)\right)_{t}$ such that $\rho_{0 T}$ is dominated and sensitive. Let $s \leq t$. For all $X \in L_{t}^{c}$, define

$$
x_{s t}(X):=\underset{g \in \mathcal{C}_{s t}^{\infty}}{\operatorname{essinf}} \tilde{\rho}_{s t}(g-X)-\underset{g \in \mathcal{C}_{s t}^{\infty}}{\operatorname{essinf}} \tilde{\rho}_{s t}(g),
$$

where $\tilde{\rho}_{s t}$ is the extension of $\rho_{\text {st }}$ to $L_{t}^{c}$ defined in Theorem 2.49 .

\section{Proposition 2.52.}

1. The operator $x_{s t}$ is well-defined on $L_{t}^{c}$ with values in $L_{s}^{c}$. It extends the operator in Definition 2.33 (see also (2.21)). Moreover,

$$
c\left(\left|x_{s t}(X)-x_{s t}(Y)\right|\right) \leq c(|X-Y|), \quad X, Y \in L_{t}^{c} .
$$

2. The operator $x_{s t}$ is convex, monotone, and satisfies the projection property. 
3. Choose $Q_{0} \in \mathcal{Q}$, then $x_{s t}$ admits the following dual representation:

$$
x_{s t}(X)=\operatorname{esssup}_{R \in \mathcal{K}}\left(E_{R}\left(X \mid \mathcal{F}_{s}\right)-\gamma_{s t}(R)\right), \quad X \in L_{t}^{c},
$$

where $\mathcal{K}$ is a set of probability measures in the dual of $L_{t}^{c}$ compact for the weak* topolology such that every $R \in \mathcal{K}$ is absolutely continuous with respect to $P$, such that the restriction of $R$ to $\mathcal{F}_{s}$ is equal to $Q_{0}$, and

$$
\gamma_{s t}(R)=\underset{Y \in L_{t}^{c}}{\operatorname{esssup}}\left(E_{R}\left(Y \mid \mathcal{F}_{s}\right)-x_{s t}(Y)\right) .
$$

Furthermore, for all $X \in L_{t}^{c}$, there exists $Q_{X} \in \mathcal{K}$ such that

$$
\left.x_{s t}(X)=E_{Q_{X}}\left(X \mid \mathcal{F}_{s}\right)-\gamma_{s t}\left(Q_{X}\right)\right) .
$$

Proof.

1. From (2.34) in Theorem 2.49 we have that

$$
\left|x_{s t}(X)-x_{s t}(Y)\right| \leq \underset{R \in \tilde{\mathcal{P}}_{s t}}{\operatorname{esssup}} E_{R}\left(|X-Y| \mid \mathcal{F}_{s}\right), \quad X, Y \in L_{t}^{c} .
$$

Then equation (2.37) follows from Lemma 2.47 item 1 and Proposition 2.48. Recall that $x_{s t}(X) \in L_{\infty}\left(\mathcal{F}_{s}\right) \subset L_{s}^{c}$, for all $X \in L_{\infty}\left(\mathcal{F}_{t}\right)$. From the continuity of $x_{s t}$ with respect to the c norm we conclude that $x_{s t}(X) \in L_{s}^{c}$, for all $X \in L_{t}^{c}$. Finally, Definition 2.51 of $x_{s t}$ extends the one given in Definition 2.33 (see also $(2.21)$ ), because $\tilde{\rho}_{s t}$ is the extension of $\rho_{s t}$ to $L_{t}^{c}$.

2. Statement 2 follows directly from the properties of $\tilde{\rho}_{s t}$, see Corollary 2.50, in the same lines as Proposition 2.39.

3. Let $Q_{0} \in \mathcal{Q}$. The map $E_{Q_{0}}\left(x_{s t}(X)\right), X \in L_{t}^{c}$, is up to a minus sign a normalised convex risk measure on $L_{t}^{c}$ with values in $\mathbb{R}$ majorized by $\sup _{Q \in \mathcal{Q}} E_{Q}(X)$. We refer to Proposition 3.1 and Theorem 3.2 in [15] to prove the existence of a set $\mathcal{K}$ of probability measures in the dual of $L_{t}^{c}$, compact for the weak* topology, such that

$$
\begin{aligned}
E_{Q_{0}}\left(x_{s t}(X)\right) & =\sup _{R \in \mathcal{K}}\left(E_{R}(X)-\gamma(R)\right) \\
& =E_{Q_{X}}(X)-\gamma\left(Q_{X}\right)
\end{aligned}
$$

for some $Q_{X} \in \mathcal{K}$ (depending on $X$ ). Thus the representations (2.38) and (2.39) follow from standard arguments, see e.g. [22].

The proof is complete. 
Now we are ready to discuss the Fatou property of a risk-indifferent evaluation, as given in Definition 2.51. This brings to the following natural definition.

Definition 2.53. For any $s \leq t$, an operator $x: L_{t}^{c} \rightarrow L_{s}^{c}$ has the Fatou property on $L_{t}^{c}$ if for any sequence $\left(X_{n}\right)_{n} \in L_{t}^{c}$, dominated in $L_{t}^{c}$, and converging $P$-a.s. to $X \in L_{t}^{c}$, we have

$$
x(X) \leq \liminf _{n \rightarrow \infty} x\left(X_{n}\right) .
$$

Here above $\left(X_{n}\right)_{n}$ dominated in $L_{t}^{c}$ means that there is $Y \in L_{t}^{c}$ such that $\left|X_{n}\right| \leq Y$ P-a.s. for all $n$.

Proposition 2.54. Set $p \in[1, \infty)$. Let $\left(\rho_{s t}\right)_{s, t}$ be a strong time-consistent, fully-dynamic risk measure on $\left(L_{p}\left(\mathcal{F}_{t}\right)\right)_{t}$ such that $\rho_{0 T}$ is dominated and sensitive. For all $s \leq t$, the operator $x_{s t}$ in Definition 2.51 has the Fatou property on $L_{t}^{c}$.

Proof. Let $\left(X_{k}\right)_{k} \in L_{t}^{c}$ be dominated in $L_{t}^{c}$ by $Y$ and converge $P$-a.s. to $X \in L_{t}^{c}$. Set $\tilde{X}_{n}:=\inf _{k \geq n} X_{k}$. The sequence $\left(\tilde{X}_{n}\right)_{n}$ is increasing, $\left|\tilde{X}_{n}\right| \leq Y$, and $X=\lim _{n \rightarrow \infty} \tilde{X}_{n} P$-a.s. From Proposition 2.52, for all $X \in L_{t}^{c}$, there is a probability measure $Q_{X}$ in the dual of $L_{t}^{c}$ such that $x_{s t}(X)=E_{Q_{X}}\left(X \mid \mathcal{F}_{s}\right)-$ $\gamma_{s t}\left(Q_{X}\right)$. Then, by the dominated convergence theorem, we obtain that

$$
\begin{aligned}
x_{s t}(X) & =\lim _{n \rightarrow \infty}\left(E_{Q_{X}}\left(\tilde{X}_{n} \mid \mathcal{F}_{s}\right)-\gamma_{s t}\left(Q_{X}\right)\right) \\
& \leq \lim _{n \rightarrow \infty} \inf _{n \geq n}\left(E_{Q_{X}}\left(X_{k} \mid \mathcal{F}_{s}\right)-\gamma_{s t}\left(Q_{X}\right)\right) .
\end{aligned}
$$

Observe that, for all $k, E_{Q_{X}}\left(X_{k} \mid \mathcal{F}_{s}\right)-\gamma_{s t}\left(Q_{X}\right) \leq x_{s t}\left(X_{k}\right)$. Then from (2.41) we get that

$$
x_{s t}(X) \leq \lim _{n \rightarrow \infty} \inf _{k \geq n} x_{s t}\left(X_{k}\right)=\liminf _{n \rightarrow \infty} x_{s t}\left(X_{n}\right) .
$$

This proves the Fatou property.

\subsection{Risk-indifference price system $\left(x_{s t}\right)_{s, t}$}

At this stage we have studied the properties of $x_{s t}$ defined on $L_{t}^{c}$ with values in $L_{s}^{c}$. We now study the time-consistency of the family $\left(x_{s t}\right)_{s, t}$.

Lemma 2.55. Let $0 \leq r \leq s \leq t$. Every $g \in \mathcal{C}_{r t}^{\infty}$ can be written $g=g_{1}+g_{2}$ for some $g_{1} \in \mathcal{C}_{r s}^{\infty}$ and $g_{2} \in \mathcal{C}_{s t}^{\infty}$.

Proof. Let $\theta \in \Theta$ such that $g \leq Y_{r t}(\theta)=Y_{r s}(\theta)+Y_{s t}(\theta)$. Let $M \in \mathbb{R}$ such that $M \leq g, M \leq Y_{r s}(\theta), M \leq Y_{s t}(\theta)$. Let

$$
g_{1}:=\inf \left(Y_{r s}(\theta),\|g\|_{\infty}-M\right) .
$$


We remark that $g_{1} \in L_{\infty}\left(\mathcal{F}_{s}\right)$ and $g_{1} \leq Y_{r s}(\theta)$. Let $g_{2}:=g-g_{1}$, clearly $g_{2} \in L_{\infty}\left(\mathcal{F}_{t}\right)$. Then we can see that $g_{2}=\sup \left(g-Y_{r s}(\theta), g-\|g\|_{\infty}+M\right)$. Hence, we obtain that $g_{2} \leq \sup \left(Y_{s t}(\theta), M\right)=Y_{s t}(\theta)$.

Lemma 2.56. Let $p \in[1, \infty]$. Let $\left(\rho_{s t}\right)_{s, t}$ be a strong time-consistent fullydynamic risk measure on $\left(L_{p}\left(\mathcal{F}_{t}\right)\right)_{t}$. Let $r \leq s \leq t$. Consider the operator $x_{s t}(X), X \in L_{p}\left(\mathcal{F}_{t}\right)$ defined $P$-a.s. as in $(2.20)$. For any $X, Y \in L_{p}\left(\mathcal{F}_{t}\right)$, assume that $x_{s t}(X) \geq x_{s t}(Y) P-$ a.s. Then $x_{r t}(X) \geq x_{r t}(Y)$.

Observe that the operator in Lemma 2.56 would be a risk-indifference price if defined on Dom $x_{s t}$, see Definition 2.33.

Proof. For the proof we deal with both $L_{p}$-convergence and $P-$ a.s. convergence. For this reason we work with an extension of the risk measure $\rho_{r s}$ from $L_{p}\left(\mathcal{F}_{s}\right)$ to the set

$$
D_{s}:=\left\{\mathcal{F}_{s}-\text { measurable } X: \exists\left(X_{n}\right)_{n} \in L_{p}\left(\mathcal{F}_{s}\right) \text { s.t. } X_{n} \uparrow X P-\text { a.s }\right\} .
$$

We define this extension as the monotone limit

$$
\bar{\rho}_{r s}(X):=\lim _{n \rightarrow \infty} \rho_{r s}\left(X_{n}\right) \quad P-a . s .
$$

First of all we show that the definition is well-posed. We consider two sequences $\left(X_{n}\right)_{n}$ and $\left(Y_{n}\right)_{n}$ in $L_{p}\left(\mathcal{F}_{s}\right)$ such that both $X_{n} \uparrow X$ and $Y_{n} \uparrow X$ $P$-a.s., where $X$ is $\mathcal{F}_{s}$-measurable. We denote $A:=\lim _{n \rightarrow \infty} \rho_{r s}\left(X_{n}\right)$ and $B:=\lim _{n \rightarrow \infty} \rho_{r s}\left(Y_{n}\right) P-$ a.s. Then for any constant $M$, we would have $X_{n} \wedge M \uparrow X \wedge M$ and also $Y_{n} \wedge M \uparrow X \wedge M$ in the $L_{p}$-convergence. Observe that $X \wedge M \in L_{p}\left(\mathcal{F}_{s}\right)$ and $\rho_{r s}$ is continuous from below, thus we have

$$
\inf _{M} \inf _{n} \rho_{r s}\left(X_{n} \wedge M\right)=\inf _{M} \rho_{r s}(X \wedge M)=\inf _{M} \inf _{n} \rho_{r s}\left(Y_{n} \wedge M\right) .
$$

Hence $A=\bar{\rho}_{r s}(X)=B$. It is also clear that $\bar{\rho}_{r s}$ is monotone and continuous $P$-a.s. from below on $D$.

We now proceed with the proof of the statement. Let $X, Y \in L_{p}\left(\mathcal{F}_{t}\right)$ such that $x_{s t}(X) \geq x_{s t}(Y)$. From (2.21) and assumption (2.19), we have that

$$
\underset{g \in \mathcal{C}_{s t}^{\infty}}{\operatorname{essinf}} \rho_{s t}(g-X) \geq \underset{g \in \mathcal{C}_{s t}^{\infty}}{\operatorname{essinf}} \rho_{s t}(g-Y) .
$$

From Lemma 2.55, any $g \in \mathcal{C}_{r t}^{\infty}$ is the sum $g=g_{1}+g_{2}$ of $g_{1} \in \mathcal{C}_{r s}^{\infty}$ and $g_{2} \in$ $\mathcal{C}_{s t}^{\infty}$. First of all from (2.42) and the lattice property of $\left\{\rho_{s t}(g-X), g \in \mathcal{C}_{s t}^{\infty}\right\}$ we observe that $g_{1}-\underset{g_{2} \in \mathcal{C}_{s t}^{\infty}}{\operatorname{essinf}} \rho_{s t}\left(g_{2}-X\right)$ belongs to $D_{s}$. Then we can apply the extension $\bar{\rho}_{r s}$ of $\rho_{r s}$ and from its monotonicity we obtain that

$$
\bar{\rho}_{r s}\left(g_{1}-\underset{g_{2} \in \mathcal{C}_{s t}^{\infty}}{\operatorname{essinf}} \rho_{s t}\left(g_{2}-X\right) \geq \bar{\rho}_{r s}\left(g_{1}-\underset{g_{2} \in \mathcal{C}_{s t}^{\infty}}{\operatorname{essinf}} \rho_{s t}\left(g_{2}-Y\right),\right.\right.
$$


for all $g_{1}$ in $\mathcal{C}_{r s}^{\infty}$. On the other hand the strong time-consistency of $\left(\rho_{s t}\right)_{s, t}$ yields

$$
\underset{g \in \mathcal{C}_{r t}^{\infty}}{\operatorname{essinf}} \rho_{r t}(g-X)=\operatorname{essinf}_{\substack{g_{1} \in \mathcal{C}_{s}^{\infty} \\ g_{2} \in \mathcal{C}_{s t}^{s}}} \rho_{r s}\left(g_{1}-\rho_{s t}\left(g_{2}-X\right)\right) .
$$

Now, let us consider a sequence $\left(h_{n}\right)_{n}$ such that $-\operatorname{essinf}_{g \in \mathcal{C}_{s t}^{\infty}} \rho_{s t}(g-X)$ is the increasing limit of $-\rho_{s t}\left(h_{n}-X\right)$. Then $-\operatorname{essinf}_{g \in \mathcal{C}_{s t}^{\infty}} \rho_{s t}(g-X)$ belongs to $D_{s}$. Then we have

$$
\bar{\rho}_{r s}\left(g_{1}-\underset{g_{2} \in \mathcal{C}_{s t}^{p}}{\operatorname{essinf}} \rho_{s t}\left(g_{2}-X\right)\right)=\lim _{n \rightarrow \infty} \rho_{r s}\left(g_{1}-\rho_{s t}\left(h_{n}-X\right)\right),
$$

from which we obtain

$$
\bar{\rho}_{r s}\left(g_{1}-\underset{g_{2} \in \mathcal{C}_{s t}^{p}}{\operatorname{essinf}} \rho_{s t}\left(g_{2}-X\right)\right)=\underset{g_{2} \in \mathcal{C}_{s t}^{\infty}}{\operatorname{essinf}} \rho_{r s}\left(g_{1}-\rho_{s t}\left(g_{2}-X\right) .\right.
$$

So from equations (2.44) and (2.43), we get

$$
\underset{g \in \mathcal{C}_{r t}^{\infty}}{\operatorname{essinf}} \rho_{r t}(g-X)=\underset{g_{1} \in \mathcal{C}_{r s}^{\infty}}{\operatorname{essinf}}\left(\bar{\rho}_{r s}\left(g_{1}-\underset{g_{2} \in \mathcal{C}_{s t}^{\infty}}{\operatorname{essinf}} \rho_{s t}\left(g_{2}-X\right)\right)\right) \geq \underset{g \in \mathcal{C}_{r t}^{\infty}}{\operatorname{essinf}} \rho_{r t}(g-Y) .
$$

The result follows.

Proposition 2.57. Fix $p \in[1, \infty]$. Let $\left(\rho_{s t}\right)_{s, t}$ be a strong time-consistent fully-dynamic risk measure on $\left(L_{p}\left(\mathcal{F}_{t}\right)\right)_{t}$. Then

$$
x_{r t}\left(x_{s t}(X)\right)=x_{r t}(X), \quad X \in L_{\infty}\left(\mathcal{F}_{t}\right) .
$$

In particular this results show that, if $p=\infty$, the family $x_{s t}$ is timeconsistent, while for $p<\infty$, the relationship is true only for essentially bounded claims.

Proof. For all $X \in L_{\infty}\left(\mathcal{F}_{t}\right)$, the value $x_{s t}(X)$ belongs to $L_{\infty}\left(\mathcal{F}_{s}\right)$, see Lemma 2.37 item 2. Then, as in [1], $x_{r t}\left(x_{s t}(X)\right)=x_{r t}(X)$ follows from the timeconsistency (Proposition 2.56) applied with $X$ and $Y=x_{s t}(X)$.

Remark 2.58. In the terminology of dynamic risk-measures, the property (2.45) is sometimes called recursive. See e.g. [1]. This is not the same as the property of strong time-consistency.

Remark 2.59. Notice that when $p<\infty$ equation (2.45) cannot be proved in $L_{p}$. Indeed it is not true in general that $x_{s t}(X)$ belongs to $L_{p}\left(\mathcal{F}_{s}\right)$ for all $X \in L_{p}\left(\mathcal{F}_{t}\right)$. This is the reason why we need to work with the extension of $x_{s t}$ to $L_{t}^{c}$. Then we know that for every $X$ in $L_{t}^{c}, x_{s t}(X)$ belongs to $L_{s}^{c} \subseteq L_{t}^{c}$. 
Theorem 2.60. Set $p \in[1, \infty)$. Let $\left(\rho_{s t}\right)_{s, t}$ be a strong time-consistent fully-dynamic risk measure on $\left(L_{p}\left(\mathcal{F}_{t}\right)\right)_{t}$ such that $\rho_{0 T}$ is dominated and sensitive. Let $x_{s t}$ be the risk-indifference price on $L_{t}^{c}$ defined as in Definition 2.51. Then $\left(x_{s t}\right)_{s, t}$ on $\left(L_{t}^{c}\right)_{t}$ is time-consistent.

For every given time horizon $t \leq T$, the price system $\left(x_{s}\right)_{s}$, defined by restriction as $x_{s}(X):=x_{s t}(X), s \leq t$, is time-consistent on the whole $L_{t}^{c}$. Namely, for all $0 \leq r \leq s \leq t$, for all $X \in L_{t}^{c}$, we have $x_{s}(X) \in L_{s}^{c}$ and

$$
x_{r}(X)=x_{r}\left(x_{s}(X)\right) .
$$

Proof. Fix $t \in[0, T]$. We obtain that

$$
x_{r t}\left(x_{s t}(X)\right)=x_{r t}(X), \quad \forall X \in L_{t}^{c},
$$

by the density of $L_{\infty}\left(\mathcal{F}_{t}\right)$ in $L_{t}^{c}$ and the uniform continuity for the c norm of $x_{s t}$ for all $s \leq t$ (Proposition 2.52) and (2.45). In turn, this gives the time-consistency of the family $\left(x_{s}\right)_{s}$, where $s \leq t$.

In the last part of this section we prove the regularity of the trajectories for the risk-indifferent price operators.

Theorem 2.61. Fix some $t \in[0, T]$. Assume that for some $Q \in \mathcal{Q}$, $\gamma_{0 t}(Q)=0$, where $\gamma_{0 t}$ is the minimal penalty for $x_{0 t}$. Then for all $X \in L_{t}^{c}$, the stochastic process $x_{s t}(X), 0 \leq s \leq t$, admits a càdlàg modification.

Proof. Notice that $\gamma_{0 t}(Q)=0$ implies that

$$
0=\sup _{X \in L_{\infty}\left(\mathcal{F}_{t}\right)}\left(E_{Q}(X)-x_{0 t}(X)\right) .
$$

For all $r \leq s \leq t$, let $y_{r s}$ be the restriction of $x_{r t}$ to $L_{s}^{c}$. From Theorem 2.60 we deduce that the family $\left(y_{r s}\right)_{0 \leq r \leq s \leq t}$ of operators on $\left(L_{s}^{c}\right)_{s}$ is strong time-consistent. Its restriction to $\left(L_{\infty}\left(\overline{\mathcal{F}}_{s}\right)\right)_{s}$ is up to a minus sign a strong time-consistent normalised dynamic risk measure on $\left(L_{\infty}\left(\mathcal{F}_{s}\right)\right)_{s}$. Making use of (2.46), the proof of Lemma 3 and of Lemma 4 in Section 3.1 of [10], applied with deterministic times, we can prove that $x_{s t}(X)$ is the limit of $x_{s_{n} t}(X)$ in $L_{1}(Q)$, for every decreasing sequence $\left(s_{n}\right)_{n}$ converging to $s$ and for every $X \in L_{\infty}\left(\mathcal{F}_{t}\right)$. We also get that $x_{r t}(X) \geq E_{Q}\left(x_{s t}(X) \mid \mathcal{F}_{r}\right)$ for all $X \in L_{\infty}\left(\mathcal{F}_{t}\right)$. Moreover, by the density of $L_{\infty}\left(\mathcal{F}_{t}\right)$ in $L_{t}^{c}$ and the uniform equicontinuity of $x_{s t}$ for the $c$ norm (equation (2.37)), we can show that, for all $X \in L_{t}^{c}$, the value $x_{s t}(X)$ is the limit of $x_{s_{n} t}(X)$ in $L_{1}(Q)$ and that $x_{s t}(X)$ is a $Q$-supermartingale for all $X \in L_{t}^{c}$. Recall that the probability measure $Q \in \mathcal{Q}$ is equivalent with $P$, then the modification theorem (see Theorem 4 page 76 in [21]) proves that, for all $X \in L_{t}^{c}$, the process $x_{s t}(X)$ admits a càdlàg modification. 
Remark 2.62. In view of the proposition above, our results are valid also with stopping times. Indeed, we could also have started our work with a fully-dynamic risk measure indexed by stopping times $\left(\rho_{\sigma, \tau}\right)_{0 \leq \sigma \leq \tau \leq T}$ as in [10] and obtain the same results of the present paper replacing deterministic times by stopping times. We stress that our framework allows to give price evaluations to all American-type financial claims.

\section{Risk-indifference prices in $L_{2}$ and no-good-deal bounds}

Good-deal bounds were suggested by Cochrane and Saa Requejo [19] in a static setting by fixing bounds on the Sharpe ratio. The idea is of identifying in this way those deals that are "too good to be true". Idea that was also considered by Bernardo and Ledoit [4] who are setting bounds on the gain-loss ratio and earlier Hodges [30] who uses a generalized Sharpe ratio derived from the negative exponential utility function, and also Cerny [16] where smooth utility functions are used to define good deals. See also [17]. In [13], starting from [19], the relationship between bounds on the Sharpe ratio and no-good-deal pricing measures was detailed providing an equivalent definition of no-good-deal bounds expressed in terms of bounds on the Radon-Nykodim derivatives. Also it was possible to define the concept of dynamic no-good-deal bounds. In this section, in view of the nature of these concepts, it is natural to work with $p=2$.

We shall use dynamic no-good-deal bounds to provide a construction of riskindifference prices in $L_{2}$. Indeed by the use of the bounds we can guarantee that, for all $s \leq t$, the risk-indifference price $x_{s t}$ (satisfying these bounds) is a well defined operator from $L_{2}\left(\mathcal{F}_{t}\right)$ to $L_{2}\left(\mathcal{F}_{s}\right)$. We shall relate this approach with the results of Section 2. Moreover, our study provides a characterisation of the risk measures $\left(\rho_{s t}\right)_{s, t}$ so that the associated risk-indifferent prices are no-good-deal prices. In the first part of this section we revise the fundamental concepts of no-good-deal bounds and provide some first results on the role of the bounds for the construction of convex operators in $L_{2}$.

\subsection{No-good-deal prices}

Definition 3.1. A probability measure $Q \sim P$ is a no-good-deal pricing measure if there are no good-deals of level $\delta>0$ under $Q$, that is, the Sharpe ratio is bounded:

$$
-\delta \leq \frac{E(X)-E_{Q}(X)}{\sqrt{\operatorname{Var}(X)}} \leq \delta,
$$

for all $X \in L_{2}\left(\mathcal{F}_{T}, P\right) \cap L_{1}\left(\mathcal{F}_{T}, Q\right)$ such that $\operatorname{Var}(X) \neq 0$. Equivalently, we can say that $Q \sim P$ is a no-good-deal pricing measure if $\frac{d Q}{d P} \in L_{2}\left(\mathcal{F}_{T}\right)$ 
satisfies

$$
E\left[\left(\frac{d Q}{d P}-1\right)^{2}\right] \leq \delta^{2}
$$

From a dynamic perspective, it is suitable to work with the following set of probability measures.

Definition 3.2. Let $s \leq t$. Let the set of probability measures on $\mathcal{F}_{t}$ be

$$
\mathfrak{Q}_{s t}:=\left\{Q \ll P: Q_{\mid \mathcal{F}_{s}}=P \text { and } \frac{d Q}{d P} \in \mathcal{D}_{s t}\right\},
$$

where

$$
\mathcal{D}_{s t}:=\left\{1+h_{s, t}: h_{s t} \in L_{2}\left(\mathcal{F}_{t}\right), E\left[h_{s, t} \mid \mathcal{F}_{s}\right]=0 E\left[h_{s t}^{2} \mid \mathcal{F}_{s}\right] \leq \delta_{s t}^{2}\right\} .
$$

Here the family of non-negative real numbers $\delta_{s t}, s, t \in[0, T]: s \leq t$, satisfies the condition for all $r \leq s \leq t$ :

$$
\left(\delta_{r s} \delta_{s t}+\delta_{r s}+\delta_{s t}\right)=\delta_{r t}
$$

and $\delta_{s t} \rightarrow 0, t \downarrow s$.

Remark that we can connect the bounds on the Sharpe ratio (3.1) with the one here above by choosing $\delta_{s t}:=\delta^{t-s}-1$, for some $\delta>1$. Then the following definition is given.

Definition 3.3. A probability measure $Q \sim P$ is a dynamic no-good-deal pricing measure if $\frac{d Q}{d P} \in L_{2}\left(\mathcal{F}_{T}\right)$ satisfies

$$
E\left[\left(\left(\frac{d Q}{d P}\right)_{t}\left(\frac{d Q}{d P}\right)_{s}^{-1}-1\right)^{2} \mid \mathcal{F}_{s}\right] \leq \delta_{s t}^{2},
$$

for every $s \leq t$ and constants $\delta_{s t}>0$ satisfying (3.3). Here $\left(\frac{d Q}{d P}\right)_{t}:=$ $E\left[\frac{d Q}{d P} \mid \mathcal{F}_{t}\right]$.

Corresponding to these bounds on the Radon-Nykodim derivatives, we can characterise the no-good-deal bounds on prices.

Definition 3.4. The no-good-deal bounds on prices are the sub-linear and super-linear operators here below:

$$
\begin{array}{ll}
M_{s t}(X):=\operatorname{esssup}_{Q \in \mathfrak{Q}_{s t}} E_{Q}\left[X \mid \mathcal{F}_{s}\right], & X \in L_{2}\left(\mathcal{F}_{t}\right), \\
m_{s t}(X):=\operatorname{essinf}_{Q \in \mathfrak{Q}_{s t}} E_{Q}\left[X \mid \mathcal{F}_{s}\right], & X \in L_{2}\left(\mathcal{F}_{t}\right),
\end{array}
$$

where $\mathfrak{Q}_{\text {st }}$ is given in Definition 3.2. 
Clearly, $m_{s t}(X)=-M_{s t}(-X)$, for $X \in L_{2}\left(\mathcal{F}_{t}\right)$. The properties of these operators are studied in Proposition 5.8 in [13].

Hereafter we study the representation of a general convex price operator $x_{s t}: L_{\infty}\left(\mathcal{F}_{t}\right) \longrightarrow L_{\infty}\left(\mathcal{F}_{s}\right)$ satisfying the no-good-deal bounds. This is a crucial result for the study of no-good-deal risk-indifference prices.

Proposition 3.5. Set $s \leq t$. Let $x_{s t}: L_{\infty}\left(\mathcal{F}_{t}\right) \longrightarrow L_{\infty}\left(\mathcal{F}_{s}\right)$ be a convex price operator Definition 2.1 satisfying the no-good-deal bound: for all $X \in$ $L_{\infty}\left(\mathcal{F}_{t}\right): X \geq 0$,

$$
x_{s t}(X) \leq M_{s t}(X) .
$$

Let $\gamma_{s t}$ be the minimal penalty of $x_{s t}$ on $L_{\infty}\left(\mathcal{F}_{t}\right)$. Then, for any probability measure $Q \ll P: Q_{\mid \mathcal{F}_{s}}=P$ and $E\left[\gamma_{s t}(Q)\right]<\infty$, we have that $Q \in \mathfrak{Q}_{s t}$ and the following representation holds

$$
x_{s t}(X)=\underset{Q \in Q_{s t}}{\operatorname{esssup}}\left(E_{Q}\left(X \mid \mathcal{F}_{s}\right)-\gamma_{s t}(Q)\right), \quad X \in L_{\infty}\left(\mathcal{F}_{t}\right) .
$$

Conversely, if (3.6) holds than (3.5) is satisfied.

Proof. From Proposition 1 in [10] we have that a convex price operator $x_{s t}$ defined on $L_{\infty}\left(\mathcal{F}_{t}\right)$ admits representation in the form

$$
x_{s t}(X)=\operatorname{esssup}_{\substack{Q \ll P: \mathcal{Q}_{\mid \mathcal{F}}=P \\ E\left[\gamma_{s t}(Q)\right]<\infty}}\left(E_{Q}\left(X \mid \mathcal{F}_{s}\right)-\gamma_{s t}(Q)\right), \quad X \in L_{\infty}\left(\mathcal{F}_{t}\right) .
$$

Consider the operator

$$
y_{s t}(X):=x_{s t}(X)-E\left(X \mid \mathcal{F}_{s}\right), \quad X \in L_{\infty}\left(\mathcal{F}_{t}\right) .
$$

From (3.5) and Definition 3.2, we have that, for $X \in L_{\infty}\left(\mathcal{F}_{t}\right): X \geq 0$

$$
y_{s t}(X) \leq \operatorname{esssup}_{h \in \mathcal{D}_{s t}} E\left((h-1) X \mid \mathcal{F}_{s}\right) \leq \delta_{s t}\left(E\left(X^{2} \mid \mathcal{F}_{s}\right)\right)^{1 / 2} .
$$

Hence, from (3.7) and (3.8), we obtain

$$
\operatorname{esssup}_{\substack{Q \ll P: \mathcal{Q}_{\mid \mathcal{F}_{s}}=P \\ \frac{d Q}{d P}=k}}\left(E\left((k-1) X \mid \mathcal{F}_{s}\right)-\gamma_{s t}(Q)\right)=y_{s t}(X) \leq \delta_{s t}\left(E\left(X^{2} \mid \mathcal{F}_{s}\right)\right)^{1 / 2},
$$

for $X \in L_{\infty}\left(\mathcal{F}_{t}\right): X \geq 0$. Consider any $Q \ll P$ such that $\mathcal{Q}_{\mid \mathcal{F}_{s}}=P, \frac{d Q}{d P}=k$ and $E\left[\gamma_{s t}(Q)\right]<\infty$. Then for every $\lambda>0$ we have

$$
E\left((k-1) \lambda X \mid \mathcal{F}_{s}\right)-\gamma_{s t}(Q) \leq \lambda \delta_{s t}\left(E\left(X^{2} \mid \mathcal{F}_{s}\right)\right)^{1 / 2} .
$$

Thus

$$
E\left((k-1) X \mid \mathcal{F}_{s}\right) \leq \delta_{s t}\left(E\left(X^{2} \mid \mathcal{F}_{s}\right)\right)^{1 / 2}+\frac{1}{\lambda} \gamma_{s t}(Q) .
$$

Taking $\lambda \rightarrow \infty$ we obtain that $E\left((k-1) X \mid \mathcal{F}_{s}\right) \leq \delta_{s t}\left(E\left(X^{2} \mid \mathcal{F}_{s}\right)\right)^{1 / 2}$. Hence, setting $h:=k-1$, we conclude that $E\left(h^{2} \mid \mathcal{F}_{s}\right) \leq \delta_{s t}^{2}$. That is $Q \in \mathfrak{Q}_{s t}$. Then $x_{s, t}$ admits representation (3.6). The converse is immediate. 
We remark that from the representation (3.6) we can see that the bound (3.5) is satisfied for all $X \in L_{\infty}\left(\mathcal{F}_{t}\right)$.

Corollary 3.6. Set $s \leq t$. Let $x_{s t}: L_{\infty}\left(\mathcal{F}_{t}\right) \longrightarrow L_{\infty}\left(\mathcal{F}_{s}\right)$ be a convex price operator satisfying the no-good-deal bound (3.5). Then $x_{\text {st }}$ is continuous in the $L_{2}$-norm and admits a unique extension

$$
x_{s t}: L_{2}\left(\mathcal{F}_{t}\right) \longrightarrow L_{2}\left(\mathcal{F}_{s}\right) .
$$

This extension admits the representation

$$
x_{s t}(X)=\underset{Q \in \mathfrak{Q}_{s t}}{\operatorname{esssup}}\left(E_{Q}\left(X \mid \mathcal{F}_{s}\right)-\gamma_{s t}(Q)\right), \quad X \in L_{2}\left(\mathcal{F}_{t}\right),
$$

and satisfies the no-good-deal bounds for all $X \in L_{2}\left(\mathcal{F}_{t}\right)$ :

$$
m_{s t}(X) \leq x_{s t}(X) \leq M_{s t}(X)
$$

Proof. We can see that $x_{s t}$ is continuous in the $L_{2}$-norm from (3.8). Hence the operator can be uniquely extended as a mapping from $L_{2}\left(\mathcal{F}_{t}\right)$ to $L_{2}\left(\mathcal{F}_{s}\right)$ by continuity. Moreover, the right-hand side of representation (3.6) is continuous in the $L_{2}$-norm, hence also extendable by continuity as a mapping from $L_{2}\left(\mathcal{F}_{t}\right)$ to $L_{2}\left(\mathcal{F}_{s}\right)$. Thus representation (3.9) holds. Moreover, for all $X \in L_{2}\left(\mathcal{F}_{t}\right), m_{s t}(X) \leq-x_{s t}(-X) \leq x_{s t}(X) \leq M_{s t}(X)$.

Remark 3.7. As for the Sharpe ratio in the static case, the dynamic nogood-deal bounds are defined without reference to some specific price model. In the case where the dynamics of some basic assets are given the question of no-free-lunch (and thus no-arbitrage) has been studied in [11]. It follows from Theorem 5.1 that a dynamic convex price system satisfies the no-freelunch condition if and only if it admits a dual representation with equivalent local martingale measures for the basic assets. In general, the no-good-deal bounds are not included in the no-arbitrage bounds. No-arbitrage no-gooddeal bounds can be constructed by replacing in the Definition 3.4 the set $\mathcal{Q}_{s t}$ by its intersection with the set of equivalent local martingale measures.

\subsection{Risk-indifference prices with no-good-deal bounds}

In this section we aim at finding necessary and sufficient conditions on the fully-dynamic risk measures, such that the associated risk-indifference price system satisfies the no-good-deal bounds. First of all we recall that any risk-indifference price system $\left(x_{s t}\right)_{s, t}$ on $\left(L_{\infty}\left(\mathcal{F}_{t}\right)\right)_{t}$ is a convex price system according to Definition 2.3.

Let $p=2$, we consider $\left(\rho_{s t}\right)_{s, t}$ to be a fully-dynamic risk measure on $\left(L_{2}\left(\mathcal{F}_{t}\right)\right)_{t}$ and $\left(x_{s t}\right)_{s, t}$ to be the family of operators defined as in (2.20), from Definition 2.33. For $s \leq t$, we consider the normalisation

$$
\breve{\rho}_{s t}(X):=\rho_{s t}(X)-\rho_{s t}(0), \quad X \in L_{2}\left(\mathcal{F}_{t}\right) .
$$


Then the restriction of $\breve{\rho}_{s t}$ to $L_{\infty}\left(\mathcal{F}_{t}\right)$ takes values in $L_{\infty}\left(\mathcal{F}_{s}\right)$ and the riskindifference price $\breve{x}_{s t}$ associated to $\breve{\rho}_{s t}(X)$ coincides with $x_{s t}$. In the following $\breve{\alpha}_{s t}$ denotes the minimal penalty associated to $\breve{\rho}_{s t}$ on $L_{\infty}\left(\mathcal{F}_{t}\right)$.

Theorem 3.8. Let $\left(\rho_{s t}\right)_{s, t}$ be a fully-dynamic risk measure on $\left(L_{2}\left(\mathcal{F}_{t}\right)\right)_{t}$ with $\breve{\rho}_{s t}$ and $\breve{\alpha}_{\text {st }}$ as above. The following two groups of assertions $A$ and $B$ are equivalent for all $s \leq t$ :

A1. $E\left(\underset{g \in \mathcal{C}_{s t}^{\infty}}{\operatorname{essinf}} \rho_{s t}(g)\right) \in \mathbb{R}$.

A2. $x_{s t}(X)=\underset{g \in \mathcal{C}_{s t}^{\infty}}{\operatorname{essinf}} \rho_{s t}(g-X)-\underset{g \in \mathcal{C}_{s t}^{\infty}}{\operatorname{essinf}} \rho_{s t}(g)$ satisfies the no-good-deal bounds on $L_{\infty}\left(\mathcal{F}_{t}\right)$.

B1. For any probability measure $R \ll P$ on $\mathcal{F}_{t}, R_{\mid \mathcal{F}_{s}}=P$, such that $E\left(\breve{\alpha}_{s t}(R)\right)<\infty$. Then either

- $R \in \mathfrak{Q}_{s t} \cap I_{s t}$

- or $R \in I_{s t}^{c}$

with

$$
I_{s t}=\left\{R \ll P, R_{\mid \mathcal{F}_{s}}=P: E\left[\underset{g \in \mathcal{C}_{s t}^{\infty}}{\operatorname{esssup}} E_{R}\left(g \mid \mathcal{F}_{s}\right)\right]<\infty\right\} .
$$

B2. The set $\mathfrak{Q}_{s t} \cap I_{s t}$ is non empty and there exists $R_{0} \in \mathfrak{Q}_{s t} \cap I_{s t}$ such that $E\left(\breve{\alpha}_{s t}\left(R_{0}\right)\right)<\infty$.

Proof. First we assume that A holds and we prove assertions B.

B1) Recall $x_{s t}(X)=\breve{x}_{s t}(X), X \in L_{\infty}\left(\mathcal{F}_{t}\right)$. Let $\gamma_{s t}$ be the minimal penalty for the restriction of $x_{s t}$ to $L_{\infty}\left(\mathcal{F}_{t}\right)$. Observe that for $R \ll P, R_{\mid \mathcal{F}_{s}}=P$ :

$$
\begin{aligned}
\gamma_{s t}(R) & =\operatorname{esssup}_{X \in L_{\infty}\left(\mathcal{F}_{t}\right)}\left[E_{R}\left(X \mid \mathcal{F}_{s}\right)-x_{s t}(X)\right] \\
& =\operatorname{esssup}_{X \in L_{\infty}\left(\mathcal{F}_{t}\right)}\left[E_{R}\left(X \mid \mathcal{F}_{s}\right)-\underset{g \in \mathcal{C}_{s t}^{\infty}}{\operatorname{essinf} \breve{\rho}_{s t}}(g-X)\right]+\underset{g \in \mathcal{C}_{s t}^{\infty}}{\operatorname{essinf} \breve{\rho}_{s t}}(g) \\
& =\underset{X \in L_{\infty}\left(\mathcal{F}_{t}\right)}{\operatorname{esssup}} \operatorname{essup}\left[\mathcal{C}_{s t}^{\infty}\right. \\
& +\underset{g \in \mathcal{C}_{s t}^{\infty}}{\operatorname{essinf} \breve{\rho}_{s t}(g)} \\
& =\operatorname{esssup}_{Y \in L_{\infty}\left(\mathcal{F}_{t}\right)}\left[E_{R}\left(-Y \mid \mathcal{F}_{s}\right)-\breve{\rho}_{s t}(g-X)+E_{R}\left(g \mid \mathcal{F}_{s}\right)\right]
\end{aligned}
$$

Hence,

$$
\gamma_{s t}(R)=\breve{\alpha}_{s t}(R)+\operatorname{esssup}_{g \in \mathcal{C}_{s t}^{\infty}} E_{R}\left(g \mid \mathcal{F}_{s}\right)+\underset{g \in \mathcal{C}_{s t}^{\infty}}{\operatorname{essinf}} \breve{\rho}_{s t}(g)
$$

From (3.11) and (A1), taking $R \ll P$ on $\mathcal{F}_{t}, R_{\mid \mathcal{F}_{s}}=P$ with $E\left(\breve{\alpha}_{s t}(R)\right)<\infty$, we have that

$$
\left.E\left(\gamma_{s t}(R)\right)<\infty \Longleftrightarrow \underset{g \in \mathcal{C}_{s t}^{\infty}}{[\operatorname{esssup}} E_{R}\left(g \mid \mathcal{F}_{s}\right)\right]<\infty
$$


So either $E\left[\operatorname{esssup}_{g \in \mathcal{C}_{s t}^{\infty}} E_{R}\left(g \mid \mathcal{F}_{s}\right)\right]=\infty$, which means that $R \in I_{s t}^{c}$, or $E\left[\operatorname{esssup}_{g \in \mathcal{C}_{s t}^{\infty}} E_{R}\left(g \mid \mathcal{F}_{s}\right)\right]<\infty$, and $R \in I_{s t}$. In this case, from (3.12), assumption A2 and Proposition 3.5, we conclude that $R \in \mathfrak{Q}_{s t}$.

B2) From the representation (3.7), there exists a probability measure $R_{0}$ such that $E\left(\gamma_{s t}\left(R_{0}\right)\right)<\infty$. The statement follows from the same arguments as above.

Now the converse, assume that B holds and we prove assertions A.

A1) Take $R_{0} \in \mathfrak{Q}_{s t} \cap I_{s t}$ such that $E\left(\breve{\alpha}_{s t}\left(R_{0}\right)\right)<\infty$. Then

$$
\begin{aligned}
E\left[\underset{g \in \mathcal{C}_{s t}^{\infty}}{\operatorname{essinf}} \breve{\rho}_{s t}(g)\right] & \geq E\left[\underset{g \in \mathcal{C}_{s t}^{\infty}}{\operatorname{essinf}} E_{R_{0}}\left(-g \mid \mathcal{F}_{s}\right)\right]-E\left(\breve{\alpha}_{s t}\left(R_{0}\right)\right) \\
& \left.=-E\left[\underset{g \in \mathcal{C}_{s t}^{\infty}}{\operatorname{esssup}} E_{R_{0}}\left(g \mid \mathcal{F}_{s}\right)\right]\right]-E\left(\breve{\alpha}_{s t}\left(R_{0}\right)\right)>-\infty
\end{aligned}
$$

A2) Consider $R \ll P$ on $\mathcal{F}_{t}, R_{\mid \mathcal{F}_{s}}=P$, such that $E\left(\gamma_{s t}(R)\right)<\infty$. Note that $\breve{\alpha}_{s t}(R) \geq-\breve{\rho}_{s t}(0)=0$, and $\left.\operatorname{esssup}_{g \in \mathcal{C}_{s t}^{\infty}} E_{R}\left(g \mid \mathcal{F}_{s}\right)\right] \geq 0$. From (3.11), taking expectation, we can see that $E\left(\breve{\alpha}_{s t}(R)\right)<\infty$ and $E\left[\operatorname{esssup}_{g \in \mathcal{C}_{s t}^{\infty}} E_{R}\left(g \mid \mathcal{F}_{s}\right)\right]<$ $\infty$. From assumption $\mathrm{B} 1$, we have $R \in \mathfrak{Q}_{s t}$. Then we have

$$
x_{s t}(X)=\operatorname{esssup}_{R \in \mathfrak{Q}_{s t}}\left[E_{R}\left(X \mid \mathcal{F}_{s}\right)-\gamma_{s t}(R)\right], \quad X \in L_{\infty}\left(\mathcal{F}_{t}\right)
$$

and $x_{s t}$ satisfies the no-good-deal bounds on $L_{\infty}\left(\mathcal{F}_{t}\right)$.

Moreover, we have the following result.

Corollary 3.9. Let $\left(\rho_{s t}\right)_{s, t}$ be a fully-dynamic risk measure on $\left(L_{2}\left(\mathcal{F}_{t}\right)\right)_{t}$ such that $E\left(\operatorname{essinf}_{g \in \mathcal{C}_{s t}^{\infty}} \rho_{s t}(g)\right) \in \mathbb{R}$. Assume that the operator $x_{s t}(X), X \in$ $L_{\infty}\left(\mathcal{F}_{t}\right)$, as in $(2.20)$ satisfies the no-good-deal bounds on $L^{\infty}\left(\mathcal{F}_{t}\right)$. Then $x_{s t}$ has a unique continuous extension

$$
x_{s t}^{n g d}: L_{2}\left(\mathcal{F}_{t}\right) \longrightarrow L_{2}\left(\mathcal{F}_{s}\right)
$$

satisfying the no-good-deal bounds on $L_{2}\left(\mathcal{F}_{t}\right)$. Furthermore, this extension admits the representation

$$
x_{s t}^{n g d}(X)=\operatorname{esssup}_{Q \in \mathfrak{Q}_{s t} \cap I_{s t}}\left(E_{Q}\left(X \mid \mathcal{F}_{s}\right)-\gamma_{s t}(Q)\right), \quad X \in L_{2}\left(\mathcal{F}_{t}\right) .
$$

Proof. The extension follows from Corollary 3.6. The representation follows from (3.9) in Corollary 3.6 and the arguments of the proof of Theorem 3.8 .

We remark that we do not know whether the extension $x_{s t}^{n g d}$ is also a riskindifferent price. We recall that in Section 2 we had assumed that the fullydynamic risk measure $\left(\rho_{s t}\right)_{s, t}$ is strong time-consistent and $\rho_{0 T}$ is dominated and sensitive. With this, starting from $x_{s t}$ on $L_{\infty}\left(\mathcal{F}_{t}\right)$ we obtained a riskindifferent extension:

$$
x_{s t}^{r i}: L_{t}^{c} \longrightarrow L_{s}^{c}
$$


where $L_{2}\left(\mathcal{F}_{t}\right) \subseteq L_{t}^{c}$. See Definition 2.51 and Theorem 2.49. Now we study the relations between these two extensions.

Theorem 3.10. Let $\left(\rho_{s t}\right)_{s, t}$ be a strong time-consistent fully-dynamic convex risk measure such that hypotheses $B$ in Theorem 3.8 are satisfied. Assume $\rho_{0 T}$ is dominated (for a constant $K>0$ ) and sensitive. Then

$$
x_{s t}^{r i}(X)=x_{s t}^{n d g}(X) \quad P-a . s ., \quad X \in L_{2}\left(\mathcal{F}_{t}\right),
$$

and $x_{s t}^{r i}(X) \in L_{2}\left(\mathcal{F}_{s}\right), X \in L_{2}\left(\mathcal{F}_{t}\right)$.

Proof. From Proposition 2.52 item 1 and the definition of the semi-norm $c$, we have that

$$
c\left(\left|x_{s t}^{r i}(X)-x_{s t}^{r i}(Y)\right|\right) \leq c(|X-Y|) \leq K\|X-Y\|_{2},
$$

for all $X, Y \in L_{2}\left(\mathcal{F}_{t}\right)$. Then $x_{s t}^{r i}$ is continuous for the $L_{2}$-norm and we can conclude $x_{s t}^{r i}(X)=x_{s t}^{n d g}(X) P-$ a.s. and then also $x_{s t}^{r i}(X) \in L_{2}\left(\mathcal{F}_{s}\right)$, for all $X \in L_{2}\left(\mathcal{F}_{t}\right)$.

\section{Conclusions.}

In this paper we study risk-indifference pricing for financial claims from a dynamic point of view. Our setting is kept free from the particular choice of underlying price dynamics and we allow for the possibility of modelling changes of risk evaluations by the use of a fully-dynamic risk measure to derive the risk-indifference prices.

Our results show that, if the risk-indifference price operators $\left(x_{s t}\right)_{s, t}$ are in $\left(L_{\infty}\left(\mathcal{F}_{t}\right)\right)_{t}$, then they constitute a convex price system. However, $\left(L_{\infty}\left(\mathcal{F}_{t}\right)\right)_{t}$ is sometimes too restrictive for financial modelling. When working with prices in $\left(L_{p}\left(\mathcal{F}_{t}\right)\right)_{t}$ with $p<\infty$, then we see that the risk-indifference prices $\left(x_{s t}\right)_{s, t}$ do not possess all the properties, as single operators, they do not have the Fatou property and as a family they are not time-consistent. The core issue is related to the very structure of the domain of these operators.

Then, for $p<\infty$, we resolve to extend these price operators to a better suited framework $\left(L_{t}^{c}\right)_{t}$, described using capacities. In performing this extension, it is needed to preserve the meaning of risk-indifference. So we first extend the whole family of underlying risk measures in a way that guarantees that the new extended fully-dynamic risk measure is also strong time-consistent. This, in turn, will guarantee that the extended prices $\left(\tilde{x}_{s t}\right)_{s, t}$ have all the properties of a convex price system on $\left(L_{t}^{c}\right)_{t}$.

From a modelling point of view it is however still desirable to have riskindifference prices in $\left(L_{p}\left(\mathcal{F}_{t}\right)\right)_{t}$. Then we look into a more specific economic context and we study risk-indifference prices that are also no-good-deal 
prices. Our results characterise those strong time-consistent fully-dynamic risk measures that provide no-good-deal risk-indifference prices. In this case, all the operators are framed in the $\left(L_{2}\left(\mathcal{F}_{t}\right)\right)_{t}$ setting.

Acknowledgements: We wish to thank Nicole El Karoui, Marco Frittelli, Marco Maggis, and Emanuela Rosazza-Gianin for the interesting discussions and their fruitful comments on this work. This research has benefit of the support of CMAP, Ecole Polytechnique and of the ToppForsk project STORM (274410) of the Research Council of Norway (NFR).

\section{References}

[1] B. Acciaio and I. Penner. Dynamic risk measures. Advanced Mathematical Methods for Finance, Eds. G. Di Nunno and B. Øksendal. pp. 1-34, Springer, Heidelberg, 2011.

[2] S. Albeverio, G. Di Nunno, and Yu.A. Rozanov. Price operators analysis in $L_{p}$-spaces. Acta Applicandae Mathematicae, 89, pp. 85-108, 2005.

[3] P. Barrieu and N. El Karoui. Pricing, hedging and optimally designing derivatives via minimization of risk measures, in: Rene Carmona (Eds.), Volume on Indifference Pricing, Princeton University Press, 2008.

[4] A.E. Bernardo and O. Ledoit. Gain, loss, and asset pricing. The Journal of Political Economy, 108, pp. 144-172, 2000.

[5] S. Biagini and M. Frittelli. On the extension of the Namioka-Klee theorem and on the Fatou property for risk measures. In Optimality and Risk: Modern trends in Mathematical Finance. The Kabanov Festschrift. Eds. F. Delbaen, M. Rasony, and C. Stricker. Springer, pp. 1-28, 2009.

[6] T. Bielecki, I. Cialenco, and M. Pitera. A survey of time consistency of dynamic risk measures and dynamic performance measures in discrete time: LM-measure perspective, Probab. Uncertain. Quant. Risk, 2,paper nr. 3, 2017.

[7] T. Bielecki, I. Cialenco, and M. Pitera. A unified approach to time consistency of dynamic risk measures and dynamic performance measures in discrete time. Mathematics of Operations Research 43 (1), pp. 204-221, 2018.

[8] J. Bion-Nadal. Conditional risk measure and robust representation of convex conditional risk measures. CMAP Preprint 557, 2004.

[9] J. Bion-Nadal. Dynamic risk measures: time-consistency and risk measures from BMO martingales. Finance and Stochastics, 12, pp. 219-244, 2008 . 
[10] J. Bion-Nadal. Time-consistent Dynamic Risk Processes. Stochastic Processes and their Applications 119 (2), pp. 633-654, 2009.

[11] J. Bion-Nadal. Bid-ask dynamic pricing in financial markets with transaction costs and liquidity risk. Journal of Mathematical Economics 45 (11), pp. 738-750, 2009.

[12] J. Bion-Nadal. Dynamic risk measures and path-dependent second order PDEs. In F.E. Benth and G. Di Nunno Eds. Stochastics of environmental and financial economics. Springer Proc. Math. Stat., 138, pp. 147-178. Springer, 2016.

[13] J. Bion-Nadal and G. Di Nunno. Dynamic no-good-deal pricing measures and extension theorems for linear operators on $L_{\infty}$. Finance and Stochastics 17 (3), pp. 587-613, 2013.

[14] J. Bion-Nadal and G. Di Nunno. Representation of convex operators and their static and dynamic sandwich extensions. Journal of Convex Analysis 24 (4), pp. 1375-1405, 2017.

[15] J. Bion-Nadal and M. Kervarec. Risk measuring under model uncertainty. The Annals of Applied Probability 22 (1), pp. 213-238, 2012.

[16] A. Cerny. Generalized Sharpe Ratio and costisten good-deal restrictions in a model of continuous trading. Discussion Paper SWP9902/F, Imperial College Management School.

[17] A. Cerny and S.D. Hodges. The theory of good-deal pricing in financial markets. In H. Geman, D. Madan, S. Pliska and T. Vorst Eds, Mathematical Finance - Bachelier Congress 2000, pp. 175-202. Springer, 2002.

[18] P. Cheridito, F. Delbaen, and M. Kupper. Dynamic monetary risk measure for bounded discrete-time processes. Electronic J. Prob. 11, pp. 57106, 2006.

[19] J.H. Cochrane and J. Saa Requejo. Beyond arbitrage: Good deal asset price bounds in incomplete markets. The Journal of Political Economy 108, pp. 1-22, 2000.

[20] F. Delbaen, S. Peng, and E. Rosazza Gianin. Representation of the penalty term of dynamic concave utilities. Finance Stoch. 14 (3), pp. 449-472, 2010.

[21] C. Dellacherie and P.A. Meyer. Probabilités et potentiel, Hermann Paris, 1980 (Chapter V-VIII - Théorie des martingales.); English translation: Probabilities and potential, north Holland, Amsterdam, 1982.

[22] K. Detlefsen and G. Scandolo. Conditional and dynamic convex risk measures. Finance and Stochastics 9, pp. 539-561, 2005. 
[23] G. Di Nunno and I.B. Eide. Lower and upper bounds of martingale measure densities in continuous time markets. Mathematical Finance 21, pp. $475-492,2011$.

[24] C. Doleans-Dade and P.A. Meyer. Une caractérisation de BMO. In: Lecture Notes in Mathematics, vol. 581, 1977.

[25] R.J. Elliott and T.K. Siu. A risk-based approach for pricing American options under a generalized Markov regime-switching model. Quant. Finance 11 (11), pp. 1633-1646, 2011.

[26] D. Filipovic and G. Svindland. The canonical model space for lawinvariant convex risk measures is $L^{1}$. Mathematical Finance 22, pp. 585589, 2012. Extended working paper version. No. 2, March 2008, Vienna Institute of Finance.

[27] M. Frittelli and M. Maggis. Conditional Certainty Equivalent. Int. J. Theor. Appl. Fin., 14 (1) pp 41-59, 2011.

[28] H. Föllmer and A. Schied. Stochastic Finance: An Introduction in Discrete Time. Second edition, de Gruyter studies in mathematics 27, 2002.

[29] M.R. Grasselli and T.R. Hurd. Indifference pricing and hedging for volatility derivatives. Appl. Math. Finance 14 (4), pp. 303-317, 2007.

[30] S.D. Hodges. A generalization of the Sharpe ration and its applications to valuation bounds and risk measures. FORC Preprint 98/88, University of Warwick, 1998.

[31] M. Kaina and L. Rüschendorf. On convex risk measures on $L^{p}$-spaces. Math. Meth. Oper. Res. 69, pp. 475-495, 2009.

[32] N. Kazamaki, Continuous Exponential Martingales and BMO. In: Lecture Notes in Mathematics, vol. 1579, 1994.

[33] S. Klöppel and M. Schweizer. Dynamic indifference valuation via convex risk measures. Mathematical Finance 17 (4), pp. 599-627, 2007.

[34] M. Kobylanski. Backward stochastic differential equations and partial differential equations with quadratic growth, Annals of Probability 28, $558-602,2000$.

[35] F.-B.Liebrich and G. Svindland. Model spaces for risk measures. Insurance Math. Econom. 77, pp. 150-165, 2017.

[36] M. Musiela and T. Zariphopoulou. Chapter 1. The single period binomial model. In Indifference pricing: theory and applications. Edited by R. Carmona. Princeton University Press, 2008. 
[37] M. Musiela and T. Zariphopoulou. Optimal asset allocation under forward exponential performance criteria. In Markov processes and related topics: a Festschrift for Thomas G. Kurtz, pp. 285-300, IMS Collect., 4, Inst. Math. Statist., 2008.

[38] J. Neveu. Discrete Parameter Martingales. North Holland, 1974.

[39] B. Øksendal and A. Sulem. Risk indifference pricing in jump diffusion markets. Math. Finance 19 (4), pp. 619-637, 2009.

[40] K. Owari. Maximum Lebesgue extension of monotone convex functions Journal of Functional Analysis, 266 (6), pp. 3572-3611, 2014.

[41] E. Pardoux and S. Peng. Adapted solution of a backward stochastic differential equation, Systems and Control Letters 14, 55-61, 1990.

[42] S. Peng. Backward SDE and related g-expectations In Backward Stochastic Differential Equations. Eds: N. El-Karoui and L. Mazliak. Pitman Research Notes in Mathematics Series 364 (1997).

[43] M. Xu. Risk measure pricing and hedging in incomplete markets. Annals of Finance 2 (1), pp 51-71, 2006. 